\title{
Relay Selections for Cooperative Underlay CR Systems with Energy Harvesting
}

\author{
Jia Ye, Student Member, IEEE, Zhedong Liu, Hui Zhao, Student Member, IEEE, Gaofeng Pan, Member, IEEE, \\ Qiang Ni, Senior Member, IEEE, and Mohamed-Slim Alouini, Fellow, IEEE
}

\begin{abstract}
In this work, we consider a cooperative underlay cognitive radio system in which one secondary user $(S)$ transmits its data to a secondary receiver with the assistance of multiple relays. In the considered underlay spectrum sharing scenario, the transmit power levers at $S$ and forwarding relays are adjusted simultaneously according to a given threshold interference power that the primary user can tolerate. We also consider that relays adopt threshold decode-and-forward relay scheme and harvest energy from the primary transmitter with finite energy storage capacity under time switching scheme. Three best relay selection schemes, optimal source-relay link with wireless energy harvesting (WEH), optimal relay-destination link with WEH and optimal source-relay-destination link with WEH schemes, are proposed and studied. Closed-form analytical expressions for the outage probability of these three best relay selection schemes are derived, respectively. Finally, our analytical results are verified by Monte-Carlo simulations.
\end{abstract}

Index Terms-Outage probability, relay selection, threshold decode-and-forward relay scheme, underlay cognitive radio networks, wireless energy harvesting.

\section{INTRODUCTION}

Energy harvesting $(\mathrm{EH})$ is a promising approach to tackle several problems, like tremendous energy consumption [1], unfeasible traditional recharging, additional power equipment [2]- [3]. By adopting EH, terminals could harvest energy from available and free ambient sources like solar, hydro, geothermal, optical power [4]- [5] and so forth. As radiofrequency (RF) signals have been widely adopted for cellular communications or television broadcast from day and night and from indoor and outdoor [6], Harvesting energy from RF signals under power splitting (PS) and time switching (TS) schemes have already been studied in previous works [7]- [8]. In TS protocol, receivers divide transmission time into two parts, which are used to gather energy from the source at the beginning of the transmission and transmit the information

Manuscript received September 26, 2018; revised January 242019 and March 21 2019; accepted March 27 2019. This work was supported in part by the Royal Society project under Grant IEC170324. The associate editor coordinating the review of this manuscript and approving it for publication was ***. (Corresponding author: Gaofeng Pan.)

J. Ye, was with Chongqing Key Laboratory of Nonlinear Circuits and Intelligent Information Processing, Southwest University, Chongqing, 400715, China, and she is now with King Abdullah University of Science and Technology, Thuwal 23955, Saudi Arabia.

Z. Liu, H. Zhao and M.-S. Alouini are with King Abdullah University of Science and Technology, Thuwal 23955, Saudi Arabia.

G. Pan is with Chongqing Key Laboratory of Nonlinear Circuits and Intelligent Information Processing, Southwest University, Chongqing, 400715, China. e-mail: gfpan@swu.edu.cn.

Q. Ni is with School of Computing and Communications, Lancaster University, Lancaster, LA1 4WA, U.K. during the remaining time. Under PS scheme, the receiver node splits the received RF signal into two parts: one for $\mathrm{EH}$ and the other for information decoding.

On the other hand, cognitive radio (CR) has also attracted numerous researchers due to its better spectrum utilization. Secondary users (SUs) could opportunistically access the spectrum bands owned by the licensed primary users (PUs) with one-way cooperation or full cooperation according to the design criteria. Nash bargaining game theoretic scheduling was proposed for joint channel and power allocation in CR systems [9]. Combining with $\mathrm{EH}$, a fascinating new research line on the green cognitive network was created [10]- [12]. SU can support its transmission by harnessing green, random and intermittent energy from PUs. However, there are still some challenges in the selection of parameters [13], like sensing duration, mode selection and detection in CR systems. An $\mathrm{EH}$ protocol was proposed in [14] for an underlay CR system with a single SU transmitter and multiple energy-constrained SU receivers.

Moreover, to expand the communication coverage and improve communication equality, the concept of relaying aided communication has been considered to be integrated into various areas, like visible light systems [15], simultaneous wireless information and power transfer (SWIPT) systems [16]. Recently, based on the advantages of the three concepts mentioned above, some researchers introduced cooperative communication and EH to CR networks [17]- [22] which leads to some challenges, especially the tradeoffs between data transmission, cooperative transmission and the time durations of EH. The authors in [17] analyzed the impact of the energy queue arrival rate at PU, relaying queue capacity and the number of relays on SU's throughput. A comparative analysis of energy-assisted decode-and-forward (DF) and the conventional relaying protocols in SWIPT cognitive framework was conducted in [18]. [19] proposed a new EH protocol for an underlay $\mathrm{CR}$ relay network with multiple PU transceivers while SUs could harvest energy from the primary system. A simple improved TS protocol with the consideration that EH relay must be sufficiently charged under a two-hop underlay CR relay network was proposed by [20]. The authors of [21] evaluated the performance of an underlay CR EH DF relaying communication system with multiple SUs and a primary destination. The joint power allocation and route selection in a multiple hop CR system were studied in [22], while considering DF relays aiding the connection between SUs.

Actually, relay selection (RS) has been proved as an efficient way to improve the diversity gain achieved at the destination 
and to simplify the implementation of the system, while making use of the fact that various copies of the same transmitted signal are available from independent fading paths at the destination. [23] and [24] investigated the outage performance of a CR system with opportunistic relays selection scheme, while considering that the source and relays are able to harvest energy, respectively. More specifically, various best RS schemes have been studied for common cooperative networks or the collaborative networks with wireless power transferring, under which the relay candidate with the optimal relay-destination link (ORDL), the optimal source-relay link (OSRL) or the optimal source-relay-destination link (OSRDL) is chosen as the relay, that is, ORDL, OSRL and OSRDL schemes [25]- [27].

Therefore, one can see that relay schemes have not been fully investigated in cooperative $\mathrm{CR}$ systems with wireless energy harvesting (WEH) and most of the existing works only studied traditional RS schemes. [23] and [24] only considered the traditional OSRDL relay selection scheme and wireless powered devices in CR scenarios. Moreover, infinite energy storage size is considered at the relays, leading to unpractical results. Motivated by the above observations and compared with these previous works, in this paper we investigate three best RS schemes, namely, OSRL, ORDL and OSRDL schemes combining with threshold DF scheme and WEH relays equipped with finite energy storage capacity in underlay CR systems. We will show that threshold DF scheme can improve the system performance in some degrees and can also weaken the system performance by setting a high threshold which decreases the diversity gain of the overall system. The main contributions of this paper are summarized as follows:

- We derive an exact expression for the outage probability (OP) of each $S-R_{n}$ link and $R_{m}-D(1 \leq m \leq M \leq$ $N)$ link in the cognitive network, while subjecting to the power constraints at PU-receiver (PU-RX).

- We characterize the distribution of the number of the available relay candidates under threshold relay scheme.

- We respectively derive the exact expressions for the OP under OSRL-WEH, ORDL-WEH and OSRDL-WEH schemes, and compare their performance via simulations.

The rest of this paper is organized as follows. In Section II, the considered cooperative CR system with WEH is presented. In Section III, the outage performance for single source-torelay link and relay-to-destination link is studied. In Section IV, threshold DF relay scheme, OSRL-WEH, ORDL-WEH and OSRDL-WEH schemes are proposed and discussed. The closed-form analytical expressions for the outage performance of the considered system with three different RS schemes are derived. In Section V, numerical results for the OP under three RS schemes are presented and discussed. Finally, we conclude the paper with some remarks in Section VI.

\section{SYSTEM MODEL}

In this paper, we consider a cooperative underlay cognitive spectrum sharing model, where the secondary network is allowed to utilize the same spectrum licensed to the primary network. The secondary cooperation network consists of $S$,

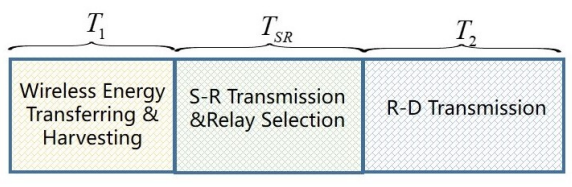

Fig. 1: Information transmission process

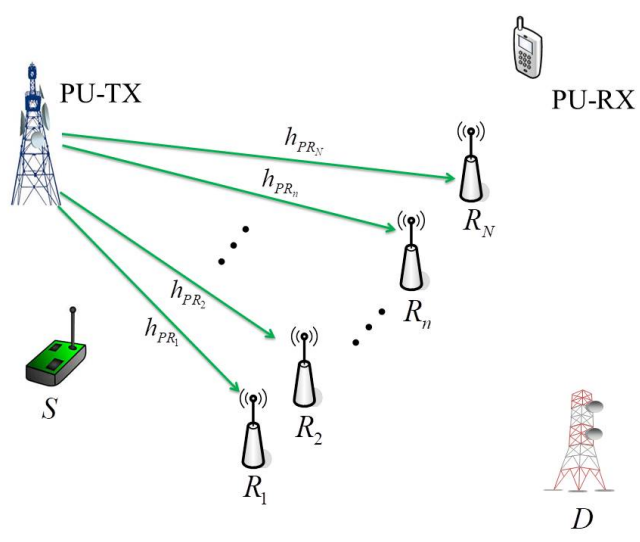

(a) Wireless energy transferring.

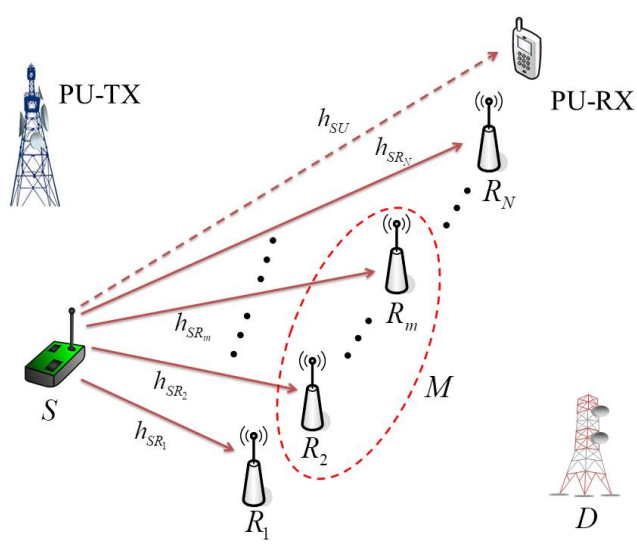

(b) Data transmission over $S-R_{n}$ link.

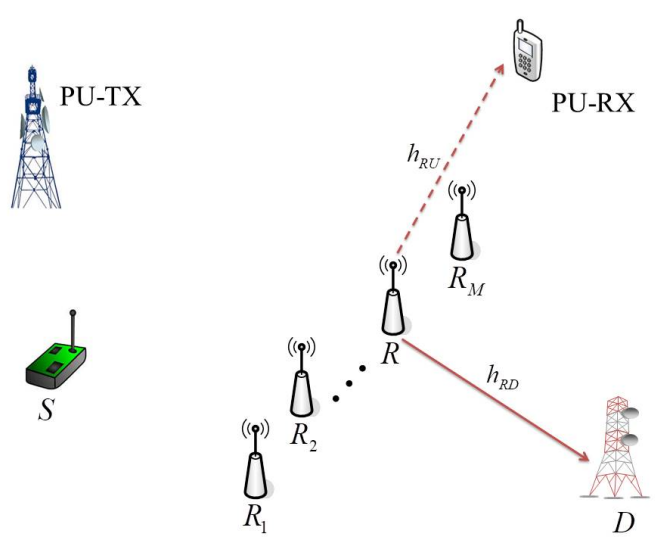

(c) Data transmission over $R-D$ link.

Fig. 2: Cooperative system model

$D$ and multiple relay nodes $\left(R_{n}, 1 \leq n \leq N, N \geq 1\right)$. It is assumed that each terminal has a single antenna and operates in a half-duplex mode. A conditional relay scheme is considered in this work: $S-R$ link based threshold DF 
scheme. The peak interference power from $S$ and all relays, which can be tolerated by PU-RX, is $I_{0}$. In this work, we considered three RS schemes to select the best relay $(R)$ to aid the information delivery from $S$ to $D$.

The information transmission process can be roughly divided into three phases, as shown in Fig. 1: 1) PU-transmitter (PU-TX) broadcasts energy via wireless signals to charge all relay candidates equipped with finite energy storage size, as shown in Fig. 2(a); 2) $S$ broadcasts the information to each relay in time slot $T_{S R}$. Under $S-R_{n}$ link based threshold DF scheme, the instantaneous received SNR of each $S-R_{n}$ is an indication of the reliability of the relaying transmission over the first hop. If the received SNR is larger than a predefined threshold $\left(\gamma_{0}\right)$, the probability of an error at the relay is small. Hence the selected relays $R_{m},(m=0,1,2, \ldots, M \leq N)$ are able to forward the signal after recoding ${ }^{1}$. Then, $R_{m}$ participate in the optimal RS process to select the best relay $R ; 3$ ) Finally, $R$ transmits the recoded information to $D$ by using the harvested energy in the first stage, while the other relays remain silent.

We here consider three kinds of RS schemes in this work:

1) OSRL-WEH: $S$ delivers a pilot signal over $S-R_{m}$ link to all relay candidates and all relay candidates feedback the information of the received SNR to $S$, and then $S$ selects the candidate with the best $S-R$ link.

2) ORDL-WEH: All relay candidates deliver a pilot signal over $R_{m}-D$ link to $D$ to let $D$ select the candidate with the best $R-D$ link.

3) OSRDL-WEH: A pilot signal is delivered over $S-R_{m}$ link from $S$ to all relay candidates and all relay candidates send the information of their received SNR and another pilot signal to $D$ to let $D$ find the candidate with the best $S-R-D$ link.

Under these three schemes, in the final step of RS process, $D$ broadcasts the selection result to all relay candidates. In the following, we denote PU-TX and PU-RX as $T$ and $U$ respectively to improve readability.

In this work, we assume that all links experience path loss with exponent $\beta$ and independent and identically Rayleigh fading ${ }^{2}$, namely, $\left|\mathbf{h}_{i j}\right|^{2},\left(i, j \in\left\{T, U, S, R_{n}, R_{m}, D\right\}\right)$. Thus it is easy to obtain the probability density function (PDF) and cumulative density function (CDF) of $\left|\mathbf{h}_{i j}\right|^{2}$ as

$$
\begin{gathered}
f_{\left|\mathbf{h}_{i j}\right|^{2}}(x)=\frac{1}{\bar{h}_{i j}} \exp \left(-\frac{x}{\bar{h}_{i j}}\right) \\
F_{\left|\mathbf{h}_{i j}\right|^{2}}(x)=1-\exp \left(-\frac{x}{\bar{h}_{i j}}\right),
\end{gathered}
$$

where $\bar{h}_{i j}$ is the expectation of channel power gain of $\left|\mathbf{h}_{i j}\right|^{2}$.

Then, in EH process, the energy harvested by $R_{n}$ with time slot $T_{1}$ can be written as $E_{R_{n}}=\frac{P_{T} T_{1}\left|\mathbf{h}_{T R_{n}}\right|^{2}}{d_{T R_{n}}^{\beta}}$, where $P_{T}$ is the transmit power at PU-TX and $d_{T R_{n}}$ represents the distance between PU-TX and $R_{n}$.

\footnotetext{
${ }^{1}$ When $m=0$, there is no relay can be selected. Obviously, an outage occurs under this case.

${ }^{2}$ The impact of large-scale fading on the outage performance of the considered system can be studied by varying the expectations of small-scale fading considered in this work.
}

During the information transmission process, the received signals at $R_{n}$ and $D$ are given by $y_{R_{n}}=\frac{\sqrt{P_{S R_{n}}} \mathbf{h}_{S R_{n}} s}{d_{S R_{n}}^{\beta}}+n_{R_{n}}$ and $y_{R_{m} D}=\frac{\sqrt{P_{R_{m} D}} \mathbf{h}_{R_{m} D} s_{R_{m}}}{d_{R_{m} D}^{\beta}}+n_{D}$, respectively, where $s$ and $s_{R_{m}}$ denote the transmitted symbols from $S$ and $R_{m}, P_{i j}$, ( $i \in\left\{S, R_{m}\right\}, j \in\left\{R_{n}, D\right\}$ ) is the transmit power used for the information delivery from node $i$ to node $j, d_{S R_{n}}$ and $d_{R_{m} D}$ represents the distance between $S$ and $R_{n}, R_{m}$ and $D$, respectively. $n_{i},\left(i \in\left\{R_{n}, D\right\}\right)$ denotes the independent complex Gaussian noise at $R_{n}$ and $D$. In this work, to simplify the analysis, we assume that $n_{i}$ is with the zero mean and the same variance, $N_{0}$.

Under the spectrum sharing mode, the transmit power at $S$ that subjects to the interference constraint suffered by PURX can be denoted as $P_{S R_{n}}=\min \left\{\frac{d_{S U}^{\beta} I_{0}}{\left|\mathbf{h}_{S U}\right|^{2}}, P_{S}\right\}$, where $I_{0}$ denotes the peak interference from $S$ or $R$ which PU-RX can tolerate, and $d_{S U}$ represents the distance between $S$ and PURX. Under underlay scheme, the interference power received at PU-RX must be within $I_{0}$, such that the maximum transmit power at $S$ should be $\frac{d_{S U}^{\beta} I_{0}}{\left|\mathbf{h}_{S U}\right|^{2}}$ and $P_{S}$ is the available transmit power at $S$.

Considering the limited energy storage size $B_{m}$ at $R_{m}$, we can also obtain the transmit power at $R_{m}$ as $P_{R_{m} D}=$ $\min \left\{\frac{E_{R_{m}}}{T_{2}}, \frac{B_{m}}{T_{2}}, \frac{d_{R_{m} U}^{\beta} I_{0}}{\left|\mathbf{h}_{R_{m} U}\right|^{2}}\right\}$, where $T_{2}$ is the transmission time slot from relays to $D, d_{R_{m} U}$ represents the distance between $R_{m}$ and PU-RX. $\frac{B_{m}}{T_{2}}$ is the maximum transmit power available at $R_{n}$, which is defined by its capacity, $\frac{E_{R_{m}}}{T_{2}}$ denotes the transmit power coming from all energy harvested during the first time slot and $\frac{d_{R_{m} U}^{\beta} I_{0}}{\left|\mathbf{h}_{R_{m} U}\right|^{2}}$ is the maximum transmit power at $R_{m}$ under underlay spectrum sharing mode.

Therefore, the received SNR at $R_{n}$ and $D$ can be written as $\gamma_{S R_{n}}=\frac{P_{S R_{n}}\left|\mathbf{h}_{S R_{n}}\right|^{2}}{d_{S R_{n}}^{\beta} N_{0}}$ and $\gamma_{R_{m} D}=\frac{P_{R_{m} D}\left|\mathbf{h}_{R_{m} D}\right|^{2}}{d_{R_{m} D}^{\beta} N_{0}}$, respectively.

\section{OUTAGE EVENTS OVER EACH HOP}

The outage probabilities for $S-R_{n}$ and $R_{m}-D$ links will be discussed in this section.

\section{A. OP over $S-R_{n}$ Link}

In this work, the OP for $S-R_{n}$ is defined as the probability that the instantaneous received SNR is below a threshold SNR $\gamma_{t h}$. Thus, when $P_{S} \geq \frac{d_{S U}^{B} I_{0}}{\left|\mathbf{h}_{S U}\right|^{2}}$, the transmit power over $S-R_{n}$ link is $P_{S R_{n}}=\frac{d_{S U}^{\beta} I_{0}}{\left|\mathbf{h}_{S U}\right|^{2}}$, the received SNR can be re-expressed as $\gamma_{R 1}=\frac{d_{S U}^{\beta} I_{0}\left|\mathbf{h}_{S R_{n}}\right|^{2}}{\left|\mathbf{h}_{S U}\right|^{2} d_{S R_{n}}^{\beta} N_{0}}$.

When $P_{S}<\frac{d_{S U}^{\beta} I_{0}}{\left|\mathbf{h}_{S U}\right|^{2}}$, the transmit power over $S-R_{n}$ link is $P_{S R_{n}}=P_{S}$. Then, the received SNR at $R_{n}$ can be reexpressed as $\gamma_{R 2}=\frac{P_{S}\left|\mathbf{h}_{S R_{n}}\right|^{2}}{d_{S R_{n}}^{\beta} N_{0}}$.

To simplify the calculation, we denote $\lambda_{R 1}=\frac{d_{S U}^{\beta} I_{0}}{d_{S R_{n}}^{\beta} N_{0}}$ and $\lambda_{R 2}=\frac{P_{S}}{d_{S R_{n}}^{\beta} N_{0}}$. Thus, the OP over $S-R_{n}$ link can be obtained 
as

$$
\begin{aligned}
P_{S R_{n}}\left(\gamma_{t h}\right) & =\operatorname{Pr}\left\{\gamma_{S R_{n}} \leq \gamma_{t h}\right\} \\
& =\operatorname{Pr}\left\{\frac{P_{S R_{n}}\left|\mathbf{h}_{S R_{n}}\right|^{2}}{d_{S R_{n}}^{\beta} N_{0}} \leq \gamma_{t h}\right\} \\
& =\operatorname{Pr}\left\{\frac{\min \left\{\frac{d_{S U}^{\beta} I_{0}}{\left|\mathbf{h}_{S U}\right|^{2}}, P_{S}\right\}\left|\mathbf{h}_{S R_{n}}\right|^{2}}{d_{S R_{n}}^{\beta} N_{0}} \leq \gamma_{t h}\right\} \\
& =\operatorname{Pr}\left\{\min \left\{\gamma_{R 1}, \gamma_{R 2}\right\} \leq \gamma_{t h}\right\} \\
& =1-\operatorname{Pr}\left\{\min \left\{\gamma_{R 1}, \gamma_{R 2}\right\}>\gamma_{t h}\right\} \\
& =1-\operatorname{Pr}\left\{\gamma_{R 1}>\gamma_{t h}, \gamma_{R 2}>\gamma_{t h}\right\} .
\end{aligned}
$$

In the following, we will calculate the joint PDF and CDF of $\gamma_{R 1}$ and $\gamma_{R 2}$. We set $X_{R 1}=\left|\mathbf{h}_{S U}\right|^{2}$ and $X_{R 2}=\left|\mathbf{h}_{S R_{n}}\right|^{2}$. Thus, $\gamma_{R 1}$ and $\gamma_{R 2}$ can be expressed by $X_{R 1}$ and $X_{R 2}$ as $\gamma_{R 1}=\frac{\lambda_{R 1} X_{R 2}}{X_{R 1}}$ and $\gamma_{R 2}=\lambda_{R 2} X_{R 2}$. Then, $X_{R 1}$ and $X_{R 2}$ can be re-expressed by $\gamma_{R 1}$ and $\gamma_{R 2}$ as $X_{R 1}=\frac{\lambda_{R 1} \gamma_{R 2}}{\lambda_{R 2} \gamma_{R 1}}$ and $X_{R 2}=\frac{\gamma_{R 2}}{\lambda_{R 2}}$. Then, we can form the Jacobian matrix as

$$
D_{\gamma_{R}}=\left[\begin{array}{ll}
\frac{\partial X_{R 1}}{\partial \gamma_{R 1}} & \frac{\partial X_{R 1}}{\partial \gamma_{R 2}} \\
\frac{\partial X_{R 2}}{\partial \gamma_{R 1}} & \frac{\partial X_{R 2}}{\partial \gamma_{R 2}}
\end{array}\right]=\left[\begin{array}{cc}
-\frac{\lambda_{R 1} \gamma_{R 2}}{\lambda_{R 2} \gamma_{R 1}{ }^{2}} & \frac{\lambda_{R 1}}{\lambda_{R 2} \gamma_{R 1}} \\
0 & \frac{1}{\lambda_{R 2}}
\end{array}\right] .
$$

Thus, the absolute value of the determinant of $D_{\gamma_{R}}$ is $\left|\operatorname{det} D_{\gamma_{R}}\right|=\frac{\lambda_{R 1} \gamma_{R 2}}{\lambda_{R 2}{ }^{2} \gamma_{R 1}{ }^{2}}$. Finally, as $X_{R 1}$ and $X_{R 2}$ are independent variables, it is easy to obtain the joint PDF of $\gamma_{R 1}$ and $\gamma_{R 1}$ as

$$
\begin{aligned}
f_{\gamma_{R 1}, \gamma_{R 2}}\left(\gamma_{R 1}, \gamma_{R 2}\right)= & \frac{\lambda_{R 1} \gamma_{R 2}}{\lambda_{R 2}{ }^{2} \gamma_{R 1}{ }^{2}} f_{X_{R 1}, X_{R 2}}\left(\frac{\lambda_{R 1} \gamma_{R 2}}{\lambda_{R 2} \gamma_{R 1}}, \frac{\gamma_{R 2}}{\lambda_{R 2}}\right) \\
= & \frac{\lambda_{R 1} \gamma_{R 2}}{\lambda_{R 2}{ }^{2} \gamma_{R 1}{ }^{2} \bar{h}_{S U} \bar{h}_{S R_{n}}} \exp \left(-\frac{\gamma_{R 2}}{\lambda_{R 2} \bar{h}_{S R_{n}}}\right) \\
& \times \exp \left(-\frac{\lambda_{R 1} \gamma_{R 2}}{\bar{h}_{S U} \lambda_{R 2} \gamma_{R 1}}\right) .
\end{aligned}
$$

By denoting $a_{1}=\frac{\lambda_{R 1}}{\lambda_{R 2}{ }^{2} \bar{h}_{S U} \bar{h}_{S R_{n}}}, a_{2}=\frac{\lambda_{R 1}}{h_{S U} \lambda_{R 2}}$ and $a_{3}=$ $\frac{1}{\lambda_{R 2} h_{S R_{n}}}$, one can calculate $\operatorname{Pr}\left\{\gamma_{R 1}>\gamma_{t h}, \gamma_{R 2}>\gamma_{t h}\right\}$ as

$$
\operatorname{Pr}\left\{\gamma_{R 1}>\gamma_{t h}, \gamma_{R 2}>\gamma_{t h}\right\}=\int_{\gamma_{t h}}^{\infty} \int_{\gamma_{t h}}^{\infty} f_{\gamma_{R 1}, \gamma_{R 2}}\left(\gamma_{R 1}, \gamma_{R 2}\right) d \gamma_{R 1} d \gamma_{R}
$$$$
=\int_{\gamma_{t h}}^{\infty} \int_{\gamma_{t h}}^{\infty} \frac{a_{1} \gamma_{R 2}}{\gamma_{R 1}^{2}} \exp \left(-\frac{a_{2} \gamma_{R 2}}{\gamma_{R 1}}\right) \exp \left(-a_{3} \gamma_{R 2}\right) d \gamma_{R 1} d \gamma_{R 2}
$$$$
=\int_{\gamma_{t h}}^{\infty} \frac{a_{1}}{a_{2}}\left[1-\exp \left(-\frac{a_{2} \gamma_{R 2}}{\gamma_{t h}}\right)\right] \exp \left(-a_{3} \gamma_{R 2}\right) d \gamma_{R 2}
$$$$
=\frac{a_{1}}{a_{2}} \int_{\gamma_{t h}}^{\infty} \exp \left(-a_{3} \gamma_{R 2}\right) d \gamma_{R 2}
$$$$
-\frac{a_{1}}{a_{2}} \int_{\gamma_{t h}}^{\infty} \exp \left(-\frac{a_{2}+a_{3} \gamma_{t h}}{\gamma_{t h}} \gamma_{R 2}\right) d \gamma_{R 2}
$$$$
=\frac{a_{1}}{a_{2} a_{3}} \exp \left(-a_{3} \gamma_{t h}\right)-\frac{a_{1} \gamma_{t h}}{a_{2}\left(a_{2}+a_{3} \gamma_{t h}\right)} \exp \left(-a_{2}-a_{3} \gamma_{t h}\right) \text {. }
$$

Finally, the OP over $S-R_{n}$ link can be obtained by substituting (6) into (3).

\section{B. OP over $R_{m}-D$ Link}

In this subsection, we will derive the OP over $R_{m}-D$ link. As discussed above, considering different transmit power at $R_{m}$, the received SNR at $D$ varies accordingly. When $P_{R_{m} D}=\frac{E_{R_{m}}}{T_{2}}$, the received $\mathrm{SNR}$ at $D$ can be expressed as

$$
\begin{aligned}
\gamma_{D 1} & =\frac{E_{R_{m}}\left|\mathbf{h}_{R_{m} D}\right|^{2}}{T_{2} N_{0}} \\
& =\frac{P_{T} T_{1}\left|\mathbf{h}_{T R_{m}}\right|^{2}\left|\mathbf{h}_{R_{m} D}\right|^{2}}{d_{T R_{m}}^{\beta} d_{R_{m} D}^{\beta} T_{2} N_{0}} .
\end{aligned}
$$

When the harvested energy exceeds the storage capacity and does not exceed the interference constraint, we can obtain $P_{R_{m} D}=\frac{B_{m}}{T_{2}}$ and the received SNR at $D$ can be given as $\gamma_{D 2}=\frac{B_{m}\left|\mathbf{h}_{R_{m} D}\right|^{2}}{d_{R_{m} D}^{\beta} T_{2} N_{0}}$.

Finally, the last situation is that the interference constraint is smaller than both the ones decided by the harvested energy and storage capacity. Accordingly, the transmit power at $R_{m}$ is $P_{R_{m} D}=\frac{d_{R_{m} U}^{\beta} I_{0}}{\left|\mathbf{h}_{R_{m} U}\right|^{2}}$ and the received SNR at $D$ can be obtained as $\gamma_{D 3}=\frac{d_{R_{m} U}^{\beta} I_{0}\left|\mathbf{h}_{R_{m} D}\right|^{2}}{\left|\mathbf{h}_{R_{m} U}\right|^{2} d_{R_{m} D}^{\beta} N_{0}}$.

Similarly, the OP over the $R_{m}-D$ link is also defined as the probability that the instantaneous received SNR is below a threshold SNR $\gamma_{t h}$, which can be expressed as

$$
\begin{aligned}
& P_{R_{m} D}\left(\gamma_{t h}\right)=\operatorname{Pr}\left\{\gamma_{R_{m} D} \leq \gamma_{t h}\right\} \\
& =\operatorname{Pr}\left\{\frac{P_{R_{m} D}\left|\mathbf{h}_{R_{m} D}\right|^{2}}{d_{R_{m} D}^{\beta} N_{0}} \leq \gamma_{t h}\right\} \\
& =\operatorname{Pr}\left\{\frac{\min \left\{\frac{E_{R_{m}}}{T_{2}}, \frac{B_{m}}{T_{2}}, \frac{d_{R_{m} U}^{\beta} I_{0}}{\left|\mathbf{h}_{R_{m} U}\right|^{2}}\right\}\left|\mathbf{h}_{R_{m} D}\right|^{2}}{d_{R_{m} D}^{\beta} N_{0}} \leq \gamma_{t h}\right\} \\
& =\operatorname{Pr}\left\{\min \left\{\gamma_{D 1}, \gamma_{D 2}, \gamma_{D 3}\right\} \leq \gamma_{t h}\right\} \\
& =1-\operatorname{Pr}\left\{\min \left\{\gamma_{D 1}, \gamma_{D 2}, \gamma_{D 3}\right\}>\gamma_{t h}\right\} \\
& =1-\operatorname{Pr}\left\{\gamma_{D 1}>\gamma_{t h}, \gamma_{D 2}>\gamma_{t h}, \gamma_{D 3}>\gamma_{t h}\right\} .
\end{aligned}
$$

In the following, we will calculate the joint PDFs and CDFs of $\gamma_{D 1}, \gamma_{D 2}, \gamma_{D 3}$. According to the definition of multivariate transformations, we set $X_{D 1}=\left|\mathbf{h}_{T R_{m}}\right|^{2}, X_{D 2}=\left|\mathbf{h}_{R_{m} D}\right|^{2}$ and $X_{D 3}=\left|\mathbf{h}_{R_{m} U}\right|^{2}$. To simplify the calculation, we denote $\lambda_{D 1}=\frac{P_{T} T_{1}}{d_{T R_{m}}^{\beta} d_{R_{m} D}^{\beta} T_{2} N_{0}}, \lambda_{D 2}=\frac{B_{m}}{d_{R_{m} D}^{\beta} T_{2} N_{0}}$ and $\lambda_{D 3}=$ $\frac{d_{R_{m} U}^{\beta} I_{0}}{d_{R_{m} D}^{\beta} N_{0}}$. Thus, $\gamma_{D 1}, \gamma_{D 2}, \gamma_{D 3}$ could be expressed by $X_{D 1}$, $X_{D 2}$ and $X_{D 3}$ as $\gamma_{D 1}=\lambda_{D 1} X_{D 1} X_{D 2}, \gamma_{D 2}=\lambda_{D 2} X_{D 2}$ and $\gamma_{D 3}=\frac{\lambda_{D 3} X_{D 2}}{X_{D 3}}$ accordingly. Then, $X_{D 1}, X_{D 2}$ and $X_{D 3}$ can be re-expressed by $\gamma_{D 1}, \gamma_{D 2}$ and $\gamma_{D 3}$ as $X_{D 1}=\frac{\lambda_{D 2} \gamma_{D 1}}{\lambda_{D 1} \gamma_{D 2}}$, $X_{D 2}=\frac{\gamma_{D 2}}{\lambda_{D 2}}$ and $X_{D 3}=\frac{\lambda_{D 3} \gamma_{D 2}}{\lambda_{D 2} \gamma_{D 3}}$, respectively.

Then, we can form the matrix of partial derivatives as

$$
\begin{aligned}
D_{\gamma_{D}}= & {\left[\begin{array}{lll}
\frac{\partial X_{D 1}}{\partial \gamma_{D 1}} & \frac{\partial X_{D 1}}{\partial \gamma_{D 2}} & \frac{\partial X_{D 1}}{\partial \gamma_{D 3}} \\
\frac{\partial X_{D 2}}{\partial \gamma_{1}} & \frac{\partial X_{D 2}}{\partial \gamma_{D 2}} & \frac{\partial X_{D 2}}{\partial \gamma_{D 3}} \\
\frac{\partial X_{D 3}}{\partial \gamma_{D 1}} & \frac{\partial X_{D 3}}{\partial \gamma_{D 2}} & \frac{\partial X_{D 3}}{\partial \gamma_{D 3}}
\end{array}\right] } \\
= & {\left[\begin{array}{ccc}
\frac{\lambda_{D 2}}{\lambda_{D 1} \gamma_{D 2}} & -\frac{\lambda_{D 2} \gamma_{D 1}}{\lambda_{D 1} \gamma_{D 2}{ }^{2}} & 0 \\
0 & \frac{1}{\lambda_{D 2}} & 0 \\
0 & \frac{\lambda_{D 3}}{\lambda_{D 2} \gamma_{D 3}} & -\frac{\lambda_{D 3} \gamma_{D 2}}{\lambda_{D 2} \gamma_{D 3}{ }^{2}}
\end{array}\right] . }
\end{aligned}
$$


Next, we can express the absolute value of the determinant of $D_{\gamma_{D}}$ as $\left|\operatorname{det} D_{\gamma_{D}}\right|=\frac{\lambda_{D 3}}{\lambda_{D 1} \lambda_{D 2} \gamma_{D 3}^{2}}$. Finally, the joint PDF of $\gamma_{D 1}, \gamma_{D 2}, \gamma_{D 3}$ can be presented as

$$
\begin{aligned}
& f_{\gamma_{D 1}, \gamma_{D 2}, \gamma_{D 3}}\left(\gamma_{D 1}, \gamma_{D 2}, \gamma_{D 3}\right)=\frac{\lambda_{D 3}}{\lambda_{D 1} \lambda_{D 2} \gamma_{D 3}{ }^{2}} \\
& \quad \times f_{X_{D 1}, X_{D 2}, X_{D 3}}\left(\frac{\lambda_{D 2} \gamma_{D 1}}{\lambda_{D 1} \gamma_{D 2}}, \frac{\gamma_{D 2}}{\lambda_{D 2}}, \frac{\lambda_{D 3} \gamma_{D 2}}{\lambda_{D 2} \gamma_{D 3}}\right) .
\end{aligned}
$$

Since $X_{D 1}, X_{D 2}$ and $X_{D 3}$ are independent with each other, we can re-express $f_{\gamma_{D 1}, \gamma_{D 2}, \gamma_{D 3}}\left(\gamma_{D 1}, \gamma_{D 2}, \gamma_{D 3}\right)$ as

$$
\begin{aligned}
& f_{\gamma_{D 1}, \gamma_{D 2}, \gamma_{D 3}}\left(\gamma_{D 1}, \gamma_{D 2}, \gamma_{D 3}\right)=\frac{\lambda_{D 3}}{\lambda_{D 1} \lambda_{D 2} \gamma_{D 3}{ }^{2}} \\
& \quad \times f_{X_{D 1}}\left(\frac{\lambda_{D 2} \gamma_{D 1}}{\lambda_{D 1} \gamma_{D 2}}\right) f_{X_{D 2}}\left(\frac{\gamma_{D 2}}{\lambda_{D 2}}\right) f_{X_{D 3}}\left(\frac{\lambda_{D 3} \gamma_{D 2}}{\lambda_{D 2} \gamma_{D 3}}\right) \\
& =\frac{\lambda_{D 3}}{\lambda_{D 1} \lambda_{D 2} \gamma_{D 3}{ }^{2} \bar{h}_{T R_{m}} \bar{h}_{R_{m} D} \bar{h}_{R_{m} U}} \\
& \quad \times \exp \left(-\frac{\lambda_{D 2} \gamma_{D 1}}{\bar{h}_{T R_{m}} \lambda_{D 1} \gamma_{D 2}}\right) \exp \left(-\frac{\gamma_{D 2}}{\bar{h}_{R_{m} D} \lambda_{D 2}}\right) \\
& \quad \times \exp \left(-\frac{\lambda_{D 3} \gamma_{D 2}}{\bar{h}_{R_{m} U} \lambda_{D 2} \gamma_{D 3}}\right) .
\end{aligned}
$$

To simplify the calculation, we denote $b_{1}=$ $\frac{\lambda_{D 3}}{\lambda_{D 1} \lambda_{D 2} h_{T R_{m}} h_{R_{m} D} h_{R_{m} U}}, b_{2}=\frac{\lambda_{D 2}}{h_{T R_{m}} \lambda_{D 1}}, b_{3}=\frac{1}{h_{R_{m} D \lambda_{D 2}}}$ and $b_{4}=\frac{\lambda_{D 3}}{h_{R_{m} U} \lambda_{D 2}}$. Thus, we can calculate $\operatorname{Pr}\left\{\gamma_{D 1}>\gamma_{t h}, \gamma_{D 2}>\gamma_{t h}, \gamma_{D 3}>\gamma_{t h}\right\}$ as

$$
\begin{aligned}
\operatorname{Pr} & \left\{\gamma_{D 1}>\gamma_{t h}, \gamma_{D 2}>\gamma_{t h}, \gamma_{D 3}>\gamma_{t h}\right\} \\
= & \int_{\gamma_{t h}}^{\infty} \int_{\gamma_{t h}}^{\infty} \int_{\gamma_{t h}}^{\infty} f_{\gamma_{D 1}, \gamma_{D 2}, \gamma_{D 3}}\left(\gamma_{D 1}, \gamma_{D 2}, \gamma_{D 3}\right) d \gamma_{D 1} d \gamma_{D 2} d \gamma_{D 3} \\
= & \int_{\gamma_{t h}}^{\infty} \exp \left(-b_{3} \gamma_{D 2}\right)\left[\int_{\gamma_{t h}}^{\infty} \frac{b_{1}}{\gamma_{D 3}{ }^{2}} \exp \left(-\frac{b_{4} \gamma_{D 2}}{\gamma_{D 3}}\right) d \gamma_{D 3}\right. \\
& \left.\times \int_{\gamma_{t h}}^{\infty} \exp \left(-\frac{b_{2} \gamma_{D 1}}{\gamma_{D 2}}\right) d \gamma_{D 1}\right] d \gamma_{D 2} \\
= & \int_{\gamma_{t h}}^{\infty} \frac{\gamma_{D 2}}{b_{2}} \exp \left(-\frac{b_{2} \gamma_{t h}}{\gamma_{D 2}}\right) \exp \left(-b_{3} \gamma_{D 2}\right) \\
& \times \frac{b_{1}}{b_{4} \gamma_{D 2}}\left[1-\exp \left(-b_{4} \gamma_{D 2} \gamma_{t h}\right)\right] d \gamma_{D 2} \\
= & \frac{b_{1}}{b_{4} b_{2}} \int_{\gamma_{t h}}^{\infty} \exp \left[-\left(\frac{b_{2} \gamma_{t h}}{\gamma_{D 2}}+b_{3} \gamma_{D 2}\right)\right] d \gamma_{D 2} \\
& -\frac{b_{1}}{b_{4} b_{2}} \int_{\gamma_{t h}}^{\infty} \exp \left\{-\left[\frac{b_{2} \gamma_{t h}}{\gamma_{D 2}}+\left(b_{3}+b_{4} \gamma_{t h}\right) \gamma_{D 2}\right]\right\} d \gamma_{D 2} .
\end{aligned}
$$

Using the approximated expression [28, Eq. (3.471.9)]

$$
\begin{aligned}
\int_{x_{0}}^{x_{1}} \exp \left(-a x-\frac{b}{x}\right) d x & \approx \sqrt{\frac{4 b}{a}} K_{1}(\sqrt{4 a b}) \\
& \times\left[\exp \left(-a x_{0}\right)-\exp \left(-a x_{1}\right)\right]
\end{aligned}
$$

where $K_{1}(\cdot)$ is the modified Bessel function of the second kind defined by [28, Eq. (8.407)], we can finally calculate $\operatorname{Pr}\left\{\gamma_{D 1}>\gamma_{t h}, \gamma_{D 2}>\gamma_{t h}, \gamma_{D 3}>\gamma_{t h}\right\}$ as

$$
\begin{aligned}
& \operatorname{Pr}\left\{\gamma_{D 1}>\gamma_{t h}, \gamma_{D 2}>\gamma_{t h}, \gamma_{D 3}>\gamma_{t h}\right\} \\
& =\frac{b_{1}}{b_{4} b_{2}} \sqrt{\frac{4 b_{2} \gamma_{t h}}{b_{3}}} K_{1}\left(\sqrt{4 b_{3} b_{2} \gamma_{t h}}\right) \exp \left(-b_{3} \gamma_{t h}\right) \\
& \quad-\frac{b_{1}}{b_{4} b_{2}} \sqrt{\frac{4 b_{2} \gamma_{t h}}{\left(b_{3}+b_{4} \gamma_{t h}\right)}} K_{1}\left(\sqrt{4\left(b_{3}+b_{4} \gamma_{t h}\right) b_{2} \gamma_{t h}}\right) \\
& \quad \times \exp \left[-\left(b_{3}+b_{4} \gamma_{t h}\right) \gamma_{t h}\right] .
\end{aligned}
$$

Finally, the OP over $R_{m}-D$ link can be obtained by substituting (14) into (8).

\section{OP UNDER THREE RS SCHEMES}

Given the selection criterion of the considered threshold DF relay scheme, we will introduce and study three types of RS methods for the considered dual-hop cooperative secondary network with WEH: OSRL-WEH, ORDL-WEH and OSRDLWEH schemes. The overall relay selection process could be divided into two parts, that is threshold relay selection and the best relay selection. In the threshold relay selection process, $S$ broadcasts the information to each relay candidate and selects the relays at which the received SNR is larger than a predefined threshold $\left(\gamma_{0}\right)$. This process promises the communication equality for the overall system by setting appropriate threshold values, as the spread of the decoding errors at the relay candidates can be avoided, while the data delivery over the first hop is failed. If there is no relay candidate be selected in this process, the forwarding process will fail. After choosing some relays that satisfy the forward condition and considering the channel equality and the transmit power constraint, the best relay will be selected to forward information by adopting three schemes, respectively. In this section, we will first analyze the threshold DF relaying selection scheme, as well as the OP under OSRL-WEH, ORDL-WEH and OSRDL-WEH schemes, respectively.

\section{A. Threshold DF Scheme}

Under $S-R_{n}$ link based threshold DF scheme, the instantaneous received SNR of each $S-R_{n}$ link is an indication of the reliability of the relaying transmission over the second hop. If the received SNR is larger than a predefined threshold, $\gamma_{0}$, the probability of an error at $R_{n}$ will decrease. Hence, $R_{n}$ can forward the information from $S$ to $D$ to participate the best RS process. Otherwise, $R_{n}$ remains silent.

Although the transmit power varies at each time slot, there is only one $S$ with single transmit antenna causing to the same transmit power for any $S-R_{n}$ link. We only need to compare the instantaneous channel gain to select the relay candidates. Therefore, in this work we assume the received SNR over $S-R_{n}$ link can be expressed as $\gamma_{R_{n}}=\frac{P\left|\mathbf{h}_{S R_{n}}\right|^{2}}{d_{S R_{n}}^{\beta} N_{0}}$ with a same transmit power $P$, while we adopt the threshold DF scheme. The probability that $R_{n}$ could successfully decode the received 
signal over $S-R_{n}$ link can be expressed as

$$
\begin{aligned}
P_{F} & =\operatorname{Pr}\left\{\gamma_{R_{n}}>\gamma_{0}\right\} \\
& =1-\operatorname{Pr}\left\{\gamma_{R_{n}} \leq \gamma_{0}\right\} \\
& =\exp \left(-\lambda_{R_{n}} \gamma_{0}\right),
\end{aligned}
$$

where $\lambda_{R_{n}}=\frac{d_{S R_{n}}^{\beta} N_{0}}{P h_{S R_{n}}}$.

Assuming the number of the relays that can forward information successfully is $M$. Then, the probability for $M=i$, $(0 \leq i \leq N)$ can be denoted as

$$
\operatorname{Pr}\{M=i\}=\left(\begin{array}{l}
N \\
i
\end{array}\right) P_{F}^{i}\left(1-P_{F}\right)^{N-i} .
$$

\section{B. OSRL-WEH Scheme}

Assuming there are $M$ relay candidates satisfying the forwarding threshold, the candidate with the best $S-R_{m}$ link is selected as the forwarding relay under the OSRL-WEH scheme. Therefore, the received SNR at the best relay $(R)$ is

$$
\gamma_{S R}^{M}=\max _{m=1, \ldots, M \leq N}\left\{\gamma_{S R_{m}} \mid \gamma_{R 3}>\gamma_{0}\right\},
$$

where $\gamma_{R 3}=\frac{P\left|\mathbf{h}_{S R_{m}}\right|^{2}}{d_{S R_{m}}^{\beta} N_{0}}$. In the following, we also denote $\lambda_{R 3}=\frac{P}{d_{S R_{m}}^{\beta} N_{0}}$ to simplify the calculation.

The OP over $S-R$ link with $M$ relay candidates can be expressed as $P_{S R}^{M}\left(\gamma_{t h}\right)=\operatorname{Pr}\left\{\gamma_{S R}^{M} \leq \gamma_{t h}\right\}$. Thus, the total OP over $S-R$ link can be expressed as

$$
P_{S R}^{O S R L}\left(\gamma_{t h}\right)=\sum_{i=0}^{N} \operatorname{Pr}\{M=i\} P_{S R}^{M}\left(\gamma_{t h}\right) .
$$

However, in order to obtain the $P_{S R}^{M}\left(\gamma_{t h}\right)$, we should calculate the OP for single $S-R_{m}$ link first. It can be denoted as $P_{S R_{m}}^{\gamma_{0}}\left(\gamma_{t h}\right)$, which could be calculated as

$$
\begin{aligned}
& P_{S R_{m}}^{\gamma_{0}}\left(\gamma_{t h}\right)=\operatorname{Pr}\left\{\gamma_{S R_{m}} \leq \gamma_{t h} \mid \gamma_{R 3}>\gamma_{0}\right\} \\
& =\operatorname{Pr}\left\{\frac{\min \left\{\frac{d_{S U}^{\beta} I_{0}}{\left|\mathbf{h}_{S U}\right|^{2}}, P_{S}\right\}\left|\mathbf{h}_{S R_{m}}\right|^{2}}{N_{0}} \leq \gamma_{t h} \mid \gamma_{R 3}>\gamma_{0}\right\} \\
& =\operatorname{Pr}\left\{\min \left\{\gamma_{R 1}, \gamma_{R 2}\right\} \leq \gamma_{t h} \mid \gamma_{R 3}>\gamma_{0}\right\} \\
& =1-\operatorname{Pr}\left\{\min \left\{\gamma_{R 1}, \gamma_{R 2}\right\}>\gamma_{t h} \mid \gamma_{R 3}>\gamma_{0}\right\} \\
& =1-\operatorname{Pr}\left\{\gamma_{R 1}>\gamma_{t h}, \gamma_{R 2}>\gamma_{t h} \mid \gamma_{R 3}>\gamma_{0}\right\}
\end{aligned}
$$

where $\left|\mathbf{h}_{S R_{m}}\right|^{2}=X_{R 2}$ and $\gamma_{R 1}=\frac{\lambda_{R 1} X_{R 2}}{X_{R 1}}, \gamma_{R 2}=$ $\lambda_{R 2} X_{R 2}$. According to Bayes' theorem, we can re-expressed $\operatorname{Pr}\left\{\gamma_{R 1}>\gamma_{t h}, \gamma_{R 2}>\gamma_{t h} \mid \gamma_{R 3}>\gamma_{0}\right\}$ as

$$
\begin{aligned}
& \operatorname{Pr}\left\{\gamma_{R 1}>\gamma_{t h}, \gamma_{R 2}>\gamma_{t h} \mid \gamma_{R 3}>\gamma_{0}\right\} \\
& =\frac{\operatorname{Pr}\left\{\gamma_{R 1}>\gamma_{t h}, \gamma_{R 2}>\gamma_{t h}, \gamma_{R 3}>\gamma_{0}\right\}}{\operatorname{Pr}\left\{\gamma_{R 3}>\gamma_{0}\right\}} .
\end{aligned}
$$

According to the adopted assumptions above, we can re-express $\gamma_{R 3}=\lambda_{R 3} X_{R 2}$. Thus,
$\operatorname{Pr}\left\{\gamma_{R 1}>\gamma_{t h}, \gamma_{R 2}>\gamma_{t h}, \gamma_{R 3}>\gamma_{0}\right\}$ can be written as

$$
\begin{aligned}
& \operatorname{Pr}\left\{\gamma_{R 1}>\gamma_{t h}, \gamma_{R 2}>\gamma_{t h}, \gamma_{R 3}>\gamma_{0}\right\} \\
& =\operatorname{Pr}\left\{\frac{\lambda_{R 1} X_{R 2}}{X_{R 1}}>\gamma_{t h}, \lambda_{R 2} X_{R 2}>\gamma_{t h}, \lambda_{R 3} X_{R 2}>\gamma_{0}\right\} \\
& =\operatorname{Pr}\left\{X_{R 2}>\frac{\gamma_{t h} X_{R 1}}{\lambda_{R 1}}, X_{R 2}>\frac{\gamma_{t h}}{\lambda_{R 2}}, X_{R 2}>\frac{\gamma_{0}}{\lambda_{R 3}}\right\} \\
& =\operatorname{Pr}\left\{X_{R 2}>\max \left\{\frac{\gamma_{t h} X_{R 1}}{\lambda_{R 1}}, \frac{\gamma_{t h}}{\lambda_{R 2}}, \frac{\gamma_{0}}{\lambda_{R 3}}\right\}\right\} .
\end{aligned}
$$

Since $\frac{\gamma_{t h}}{\lambda_{R 2}}$ and $\frac{\gamma_{0}}{\lambda_{R 3}}$ are both constants, we denote $D=\max \left\{\frac{\gamma_{t h}}{\lambda_{R 2}}, \frac{\gamma_{0}}{\lambda_{R 3}}\right\}$ to simplify the calculation. Thus, $\operatorname{Pr}\left\{\gamma_{R 1}>\gamma_{t h}, \gamma_{R 2}>\gamma_{t h}, \gamma_{R 3}>\gamma_{0}\right\}$ can be re-expressed as

$$
\begin{aligned}
& \operatorname{Pr}\left\{\gamma_{R 1}>\gamma_{t h}, \gamma_{R 2}>\gamma_{t h}, \gamma_{R 3}>\gamma_{0}\right\} \\
& =\operatorname{Pr}\left\{X_{R 2}>\max \left\{\frac{\gamma_{t h} X_{R 1}}{\lambda_{R 1}}, D\right\}\right\} \\
& =1-\operatorname{Pr}\left\{X_{R 2} \leq \max \left\{\frac{\gamma_{t h} X_{R 1}}{\lambda_{R 1}}, D\right\}\right\} \\
& =1-F_{X_{R 2}}\left(\max \left\{\frac{\gamma_{t h} X_{R 1}}{\lambda_{R 1}}, D\right\}\right) \\
& =\exp \left\{-\frac{\max \left\{\frac{\gamma_{t h} X_{R 1}}{\lambda_{R 1}}, D\right\}}{\bar{h}_{S R_{m}}}\right\} .
\end{aligned}
$$

Since $\operatorname{Pr}\left\{\gamma_{R 3}>\gamma_{0}\right\}=P_{F}$, which denote the probability that $R_{m}$ could successfully decode the received signal. We can obtained $P_{S R_{m}}^{\gamma_{0}}\left(\gamma_{t h}\right)$ as

$$
P_{S R_{m}}^{\gamma_{0}}\left(\gamma_{t h}\right)=1-\frac{\exp \left\{-\frac{\max \left\{\frac{\gamma_{t h} X_{R 1}}{\lambda_{R 1}}, D\right\}}{\bar{h}_{S R_{m}}}\right\}}{P_{F}} .
$$

According to (17) and considering i.i.d Rayleigh fading channel, we can obtain $P_{S R}^{M}\left(\gamma_{t h}\right)$ as

$$
\begin{aligned}
& P_{S R}^{M}\left(\gamma_{t h}\right)=\prod_{m=1, \ldots, M \leq N}^{M} P_{S R_{m}}^{\gamma_{0}}\left(\gamma_{t h}\right) \\
& =\left[1-\frac{\exp \left\{-\frac{\max \left\{\frac{\gamma_{t h} X_{R 1}}{\lambda_{R 1}}, D\right\}}{\bar{h}_{S R_{m}}}\right\}}{P_{F}}\right]^{M} \\
& =\sum_{k=0}^{M}\left(\begin{array}{c}
M \\
k
\end{array}\right)(-1)^{k} P_{F}^{-k} \exp \left\{-\frac{k \max \left\{\frac{\gamma_{t h} X_{R 1}}{\lambda_{R 1}}, D\right\}}{\bar{h}_{S R_{m}}}\right\} .
\end{aligned}
$$

Considering the distribution of $X_{R 1}$, we can re-expressed $P_{S R}^{M}\left(\gamma_{t h}\right)$ as

$$
\begin{aligned}
P_{S R}^{M}\left(\gamma_{t h}\right)= & \sum_{k=0}^{M}\left(\begin{array}{c}
M \\
k
\end{array}\right)(-1)^{k} P_{F}^{-k} \\
& \times \underbrace{\int_{0}^{\infty} \exp \left\{-\frac{k \max \left\{\frac{\gamma_{t h} x}{\lambda_{R 1}}, D\right\}}{\bar{h}_{S R_{m}}}\right\} f_{X_{R 1}}(x) d x}_{I},
\end{aligned}
$$


where $I$ can be calculated as

$$
\begin{aligned}
I= & \int_{0}^{\lambda_{R 1} D / \gamma_{t h}} \exp \left(-\frac{k D}{\bar{h}_{S R_{m}}}\right) f_{X_{R 1}}(x) d x \\
& +\int_{\lambda_{R 1} D / \gamma_{t h}}^{\infty} \exp \left(-\frac{k \gamma_{t h} x}{\lambda_{R 1} \bar{h}_{S R_{m}}}\right) f_{X_{R 1}}(x) d x \\
= & \int_{\lambda_{R 1} D / \gamma_{t h}}^{\infty} \exp \left(-\frac{k \gamma_{t h} x}{\lambda_{R 1} \bar{h}_{S R_{m}}}\right) \frac{1}{\bar{h}_{S U}} \exp \left(-\frac{x}{\bar{h}_{S U}}\right) d x \\
& +\int_{0}^{\lambda_{R 1} D / \gamma_{t h}} \exp \left(-\frac{k D}{\bar{h}_{S R_{m}}}\right) \frac{1}{\bar{h}_{S U}} \exp \left(-\frac{x}{\bar{h}_{S U}}\right) d x \\
= & \left.\frac{D\left(k \gamma_{t h} \bar{h}_{S U}+\bar{h}_{S R_{m}} \lambda_{R 1}\right)}{\bar{h}_{S R_{m}} \bar{h}_{S U} \gamma_{t h}}\right] \\
& +\exp \left(-\frac{k D}{\bar{h}_{S U}+\bar{h}_{S R_{m}} \lambda_{R 1}} \exp \left[1-\exp \left(-\frac{\lambda_{R 1} D}{\bar{h}_{S U} \gamma_{t h}}\right)\right] .\right.
\end{aligned}
$$

Thus, one can obtain $P_{S R}^{M}\left(\gamma_{t h}\right)$ and $P_{S R}^{O S R L}\left(\gamma_{t h}\right)$ accordingly. Finally, the OP of the considered system under OSRLWEH scheme can be obtained by using (18) and (8), which can be expressed as

$$
\begin{aligned}
P_{O S R L}\left(\gamma_{t h}\right)= & 1-\left[1-P_{S R}^{O S R L}\left(\gamma_{t h}\right)\right]\left[1-P_{R_{m} D}\left(\gamma_{t h}\right)\right] \\
= & P_{S R}^{O S R L}\left(\gamma_{t h}\right)+P_{R_{m} D}\left(\gamma_{t h}\right) \\
& -P_{S R}^{O S R L}\left(\gamma_{t h}\right) P_{R_{m} D}\left(\gamma_{t h}\right) .
\end{aligned}
$$

\section{ORDL-WEH Scheme}

Similarly, assuming there are $M$ relay candidates satisfying the forwarding threshold, the candidate with the best $R_{m}$ $D$ link is selected as the forwarding relay under the ORDLWEH scheme. By assuming i.i.d Rayleigh fading channels, the received SNR of $R_{m}-D$ link is independent with $S-R$ link while the selected best forwarding relay must satisfy the threshold condition. Therefore, the received SNR at the best relay $(R)$ is

$$
\gamma_{R D}^{M}=\max _{m=1, \ldots, M \leq N}\left\{\gamma_{R_{m} D}\right\} .
$$

The OP over $R-D$ link with $M$ relay candidates can be expressed as

$$
\begin{aligned}
\operatorname{Pr}\left\{\gamma_{R D}^{M} \leq \gamma_{t h}\right\} & =\operatorname{Pr}\left\{\max _{m=1, \ldots, M}\left\{\gamma_{R_{m} D}\right\} \leq \gamma_{t h}\right\} \\
& =\prod_{m=1}^{M} P_{R_{m} D}\left(\gamma_{t h}\right),
\end{aligned}
$$

where $P_{R_{m} D}\left(\gamma_{t h}\right)$ has been obtained in (8). Thus, the total OP over $R-D$ link can be expressed as

$$
\operatorname{Pr}\left\{\gamma_{R D} \leq \gamma_{t h}\right\}=\sum_{i=0}^{N} \operatorname{Pr}\{M=i\} \prod_{m=1}^{M=i} P_{R_{m} D}\left(\gamma_{t h}\right) .
$$

By considering the received SNR of $S-R$ link of the best relay is greater than the threshold, the OP of the considered system can be expressed as

$$
\begin{aligned}
P_{O R D L}\left(\gamma_{t h}\right)= & 1-\left[1-\operatorname{Pr}\left\{\gamma_{R D} \leq \gamma_{t h}\right\}\right] \\
& \times\left[1-\operatorname{Pr}\left\{\gamma_{S R} \leq \gamma_{t h} \mid \gamma_{R 3}>\gamma_{0}\right\}\right] \\
= & \operatorname{Pr}\left\{\gamma_{R D} \leq \gamma_{t h}\right\}+\operatorname{Pr}\left\{\gamma_{S R} \leq \gamma_{t h} \mid \gamma_{R 3}>\gamma_{0}\right\} \\
& -\operatorname{Pr}\left\{\gamma_{R D} \leq \gamma_{t h}\right\} \operatorname{Pr}\left\{\gamma_{S R} \leq \gamma_{t h} \mid \gamma_{R 3}>\gamma_{0}\right\},
\end{aligned}
$$

where $\operatorname{Pr}\left\{\gamma_{S R} \leq \gamma_{t h} \mid \gamma_{R 3}>\gamma_{0}\right\}=P_{S R_{m}}^{\gamma_{0}}\left(\gamma_{t h}\right)$ obtained in (23). Thus, considering the distribution of $X_{R 1}$, we can reexpress $P_{S R_{m}}^{\gamma_{0}}\left(\gamma_{t h}\right)$ as

$$
\begin{aligned}
& P_{S R_{m}}^{\gamma_{0}}\left(\gamma_{t h}\right)=1-\int_{0}^{\infty} \frac{\exp \left\{-\frac{\max \left\{\frac{\gamma_{t h} x}{\lambda_{R 1}}, D\right\}}{\bar{h}_{S R}}\right\}}{P_{F}} f_{X_{R 1}}(x) d x \\
& =1-P_{F}{ }^{-1}\left[\int_{0}^{\lambda_{R 1} D / \gamma_{t h}} \frac{1}{\bar{h}_{S U}} \exp \left(-\frac{D}{\bar{h}_{S R}}-\frac{x}{\bar{h}_{S U}}\right) d x\right. \\
& \left.\quad+\int_{\lambda_{R 1} D / \gamma_{t h}}^{\infty} \frac{1}{\bar{h}_{S U}} \exp \left(-\frac{\gamma_{t h} x}{\lambda_{R 1} \bar{h}_{S R}}-\frac{x}{\bar{h}_{S U}}\right)\right] d x \\
& =1-P_{F}{ }^{-1}\left\{\exp \left(-\frac{D}{\bar{h}_{S R}}\right)\left[1-\exp \left(-\frac{\lambda_{R 1} D}{\bar{h}_{S U} \gamma_{t h}}\right)\right]\right. \\
& \left.+\frac{\bar{h}_{S R} \lambda_{R 1}}{\gamma_{t h} \bar{h}_{S U}+\bar{h}_{S R} \lambda_{R 1}} \exp \left[-\frac{D\left(\gamma_{t h} \bar{h}_{S U}+\bar{h}_{S R} \lambda_{R 1}\right)}{\bar{h}_{S R} \bar{h}_{S U} \gamma_{t h}}\right]\right\} .
\end{aligned}
$$

Finally, we can obtain the OP of the considered system under ORDL-WEH scheme by substituting (30) and (32) into (31).

\section{OSRDL-WEH Scheme}

Under the OSRDL-WEH scheme, the first step is to find the minimum received SNR among the two hops for each $S-R_{m}-D$ link while the received SNR of each $S-R_{m}$ link is greater than the threshold. The next step is to select the candidate with the maximum of the obtained SNRs from the first step at the relay. Thus, the SNR of the received signal at $D$ with $M$ relay candidates should satisfy

$$
\gamma_{S R D}^{M}=\max _{m=1, \ldots, M \leq N}\left\{\min \left\{\gamma_{S R_{m}} \mid \gamma_{R 3}>\gamma_{0}, \gamma_{R_{m} D}\right\}\right\} .
$$

The OP under the OSRDL-WEH scheme with $M$ relay candidates can be expressed as $P_{S R D}^{M}\left(\gamma_{t h}\right)=\operatorname{Pr}\left\{\gamma_{S R D}^{M} \leq \gamma_{t h}\right\}$. Thus, the total OP under this scheme can be expressed as

$$
P_{S R D}^{O S R D L}\left(\gamma_{t h}\right)=\sum_{i=0}^{N} \operatorname{Pr}\{M=i\} P_{S R D}^{M}\left(\gamma_{t h}\right) .
$$

In order to obtain $P_{S R D}^{M}\left(\gamma_{t h}\right)$, we need to calculate the OP for single $S-R_{m}-D$ link first. It can be denoted as $P_{S R_{m} D}\left(\gamma_{t h}\right)$, which could be calculated as

$$
\begin{aligned}
& P_{S R_{m} D}\left(\gamma_{t h}\right)=\operatorname{Pr}\left\{\min \left\{\gamma_{S R_{m}}, \gamma_{R_{m} D}\right\} \leq \gamma_{t h}\right\} \\
& =1-\left[1-P_{S R_{m}}^{\gamma_{0}}\left(\gamma_{t h}\right)\right]\left[1-P_{R_{m} D}\left(\gamma_{t h}\right)\right] .
\end{aligned}
$$




$$
\begin{aligned}
P_{S R_{m} D}\left(\gamma_{t h}\right)= & 1-\frac{\exp \left\{-\frac{\max \left\{\frac{\gamma_{t h} x_{R 1}}{\lambda_{R 1}, D}\right\}}{h_{S R_{m}}}\right\}}{P_{F}}\left\{\frac{b_{1}}{b_{4} b_{2}} \sqrt{\frac{4 b_{2} \gamma_{t h}}{b_{3}}} K_{1}\left(\sqrt{4 b_{3} b_{2} \gamma_{t h}}\right) \exp \left(-b_{3} \gamma_{t h}\right)\right. \\
& \left.-\frac{b_{1}}{b_{4} b_{2}} \sqrt{\frac{4 b_{2} \gamma_{t h}}{\left(b_{3}+b_{4} \gamma_{t h}\right)}} K_{1}\left(\sqrt{4\left(b_{3}+b_{4} \gamma_{t h}\right) b_{2} \gamma_{t h}}\right) \exp \left[-\left(b_{3}+b_{4} \gamma_{t h}\right) \gamma_{t h}\right]\right\}
\end{aligned}
$$

Thus, using (8) and (23), we can obtain $P_{S R_{m} D}\left(\gamma_{t h}\right)$ as shown on the top of next page.

According to (33) and considering i.i.d Rayleigh fading channel, $P_{S R D}^{M}\left(\gamma_{t h}\right)$ can be calculated as

$$
\begin{aligned}
P_{S R D}^{M}\left(\gamma_{t h}\right)= & \prod_{m=1}^{M}\left\{1-\left[1-P_{R_{m} D}\left(\gamma_{t h}\right)\right]\right. \\
& \left.\times P_{F}^{-1} \exp \left[-\frac{\max \left\{\frac{\gamma_{t h} X_{R 1}}{\lambda_{R 1}}, D\right\}}{\bar{h}_{S R_{m}}}\right]\right\} \\
= & \sum_{k=0}^{M}\left(\begin{array}{l}
M \\
k
\end{array}\right)(-1)^{k} P_{F}^{-k}\left(1-P_{R_{m} D}\left(\gamma_{t h}\right)\right)^{k} \\
& \times \exp \left\{-\frac{k \max \left\{\frac{\gamma_{t h} X_{R 1}}{\lambda_{R 1}}, D\right\}}{\bar{h}_{S R_{m}}}\right\}
\end{aligned}
$$

By considering the distribution of $X_{R 1}, P_{S R D}^{M}\left(\gamma_{t h}\right)$ can be re-expressed as

$$
\begin{aligned}
P_{S R D}^{M}\left(\gamma_{t h}\right)= & \sum_{k=0}^{M}\left(\begin{array}{c}
M \\
k
\end{array}\right)(-1)^{k} P_{F}^{-k}\left(1-P_{R_{m} D}\left(\gamma_{t h}\right)\right)^{k} \\
& \times \int_{0}^{\infty} \exp \left\{-\frac{k \max \left\{\frac{\gamma_{t h} x}{\lambda_{R 1}}, D\right\}}{\bar{h}_{S R_{m}}}\right\} f_{X_{R 1}}(x) d x \\
= & \sum_{k=0}^{M}\left(\begin{array}{l}
M \\
k
\end{array}\right)(-1)^{k} P_{F}{ }^{-k}\left(1-P_{R_{m} D}\left(\gamma_{t h}\right)\right)^{k} I,
\end{aligned}
$$

where $I$ was obtained in (26).

Finally, the OP of the considered system under OSRDLWEH scheme can be achieved by inserting (38) into (34).

\section{Numerical Results}

In this section, we will present some numerical results by running Monte Carlo simulation to validate our proposed analysis models of the OP for the considered system.

\section{A. OSRL-WEH Scheme}

In this subsection, the best relay is selected by OSRLWEH scheme. According to [5] and [25], the main adopted parameters are set as: $T_{1}=400 \mathrm{~s}, T_{2}=200 \mathrm{~s}, B_{R}=500$ $\mathrm{mAh} \times 3.0 \mathrm{~V}, P_{T}=15 \mathrm{~dB}, P_{S}=8 \mathrm{~dB}, I_{0}=20 \mathrm{~dB}, \gamma_{0}=0.5$ $\mathrm{dB}, \gamma_{t h}=1 \mathrm{~dB}, \bar{h}_{T R}=2 \mathrm{~dB}, \bar{h}_{S U}=8 \mathrm{~dB}, \bar{h}_{R U}=6 \mathrm{~dB}$, $\bar{h}_{R D}=10 \mathrm{~dB}, d_{T R}=d_{S R}=d_{S U}=d_{R U}=d_{R D}=1 \mathrm{~m}$, $\beta=2$ and $N_{0}=1$.

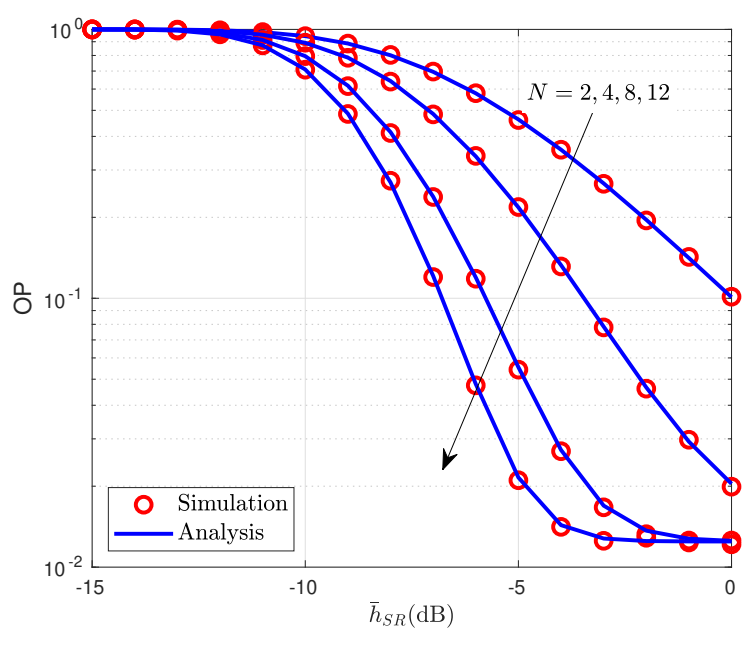

Fig. 3: OP versus $\bar{h}_{S R}$ for $P=5 \mathrm{~dB}$.

First, we show the impact of the number of relay candidates on the OP of the considered system. Fig. 3 presents the curves of the OP for various $N$. It is easy to see that OP can be improved while $N$ increases, because of the increased diversity gain. However, the improvement is more evident in the lower $N$ field since it cannot influence the OP over $R-D$ link which also leads to the lower bound of the OP of the overall system.

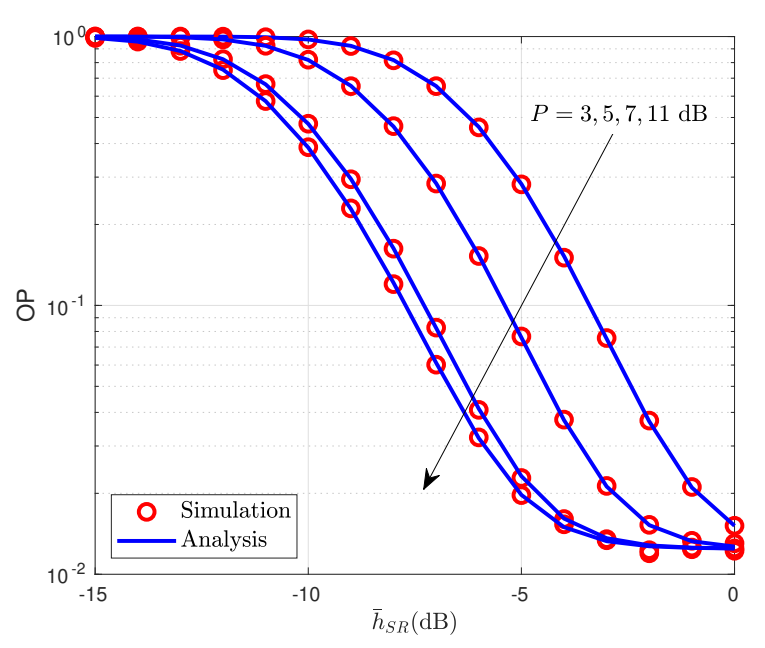

Fig. 4: OP versus $\bar{h}_{S R}$ for $N=7$.

As depicted in Fig. 4, the outage performance can be significantly improved by increasing $P$, which means more available candidates participate in the best relay selection 
process. However, the improvement is less obvious in high $P$ field since the probability that all relays are engaging the best relay selection process increases while $P$ increases. Moreover, we can also observe that there is a floor existing for each OP line in high $\bar{h}_{S R}$ region. The reason is that the outage performance over $R-D$ link mainly decides the OP of the objective system in high $\bar{h}_{S R}$ region.

\section{B. ORDL-WEH Scheme}

In this subsection, the best relay is selected by ORDLWEH scheme. As suggested in [5] and [25], the main adopted parameters are set as: $T_{1}=100 \mathrm{~s}, T_{2}=400 \mathrm{~s}, B_{R}=500$ $\mathrm{mAh} \times 3.0 \mathrm{~V}, P_{S}=18 \mathrm{~dB}, P=5 \mathrm{~dB}, I_{0}=15 \mathrm{~dB}, \gamma_{t h}=1$ $\mathrm{dB}, N=5, \bar{h}_{T R}=6 \mathrm{~dB}, \bar{h}_{R U}=5 \mathrm{~dB}, \bar{h}_{S R}=10 \mathrm{~dB}$, $\bar{h}_{R D}=10 \mathrm{~dB}, d_{T R}=d_{S R}=d_{S U}=d_{R U}=d_{R D}=1 \mathrm{~m}$, $\beta=2$ and $N_{0}=1$.

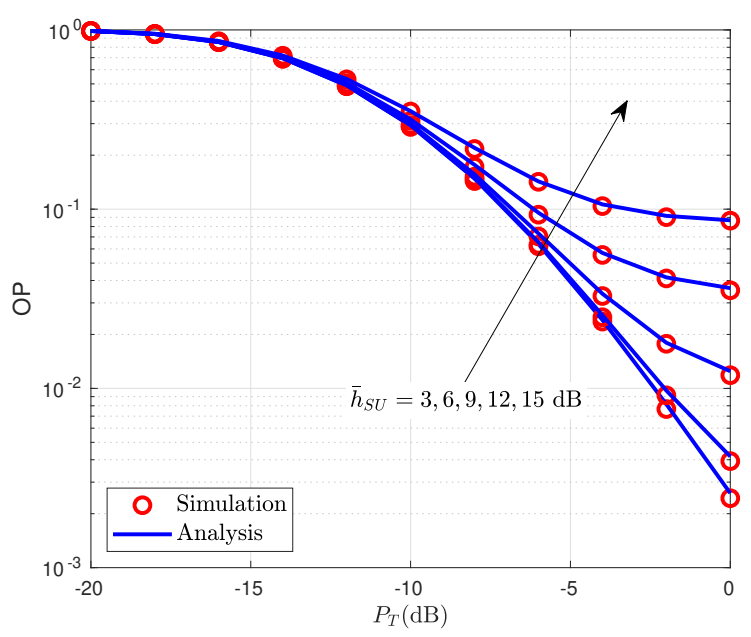

Fig. 5: OP versus $P_{T}$ for $\gamma_{0}=0.5 \mathrm{~dB}$.

As shown in Fig. 5, OP can be improved by decreasing the channel quality of $S$ to PU-RX link, which leads to the decreased received signal strength at PU-RX. Thus, the transmit power at $S$ could be improved under worse channel condition. There is nearly no influence on the low $P_{T}$ region, since the OP of $R-D$ link plays a main role in system performance.

In Fig. 6, we plot OP versus $P_{T}$ for different $\gamma_{0}$. We can observe that the OP with a low $\gamma_{0}$ outperforms the one with a high $\gamma_{0}$ in high $\gamma_{0}$ region, since only few relays can participate in the best relay selection process. However, the OP with a high $\gamma_{0}$ outperforms the one with a low $\gamma_{0}$ in low $\gamma_{0}$ region. This is because $\gamma_{0}$ could select better $S-R$ link which plays a significant role in the low bound of the OP of the overall system.

\section{OSRDL-WEH Scheme}

In this subsection, the best relay is selected by OSRDLWEH scheme. Following [5] and [25], the main adopted parameters are set as: $T_{1}=100 \mathrm{~s}, T_{2}=300 \mathrm{~s}, B_{R}=500$ $\mathrm{mAh} \times 3.0 \mathrm{~V}, P_{T}=12 \mathrm{~dB}, P_{S}=10 \mathrm{~dB}, P=5 \mathrm{~dB}, I_{0}=10$

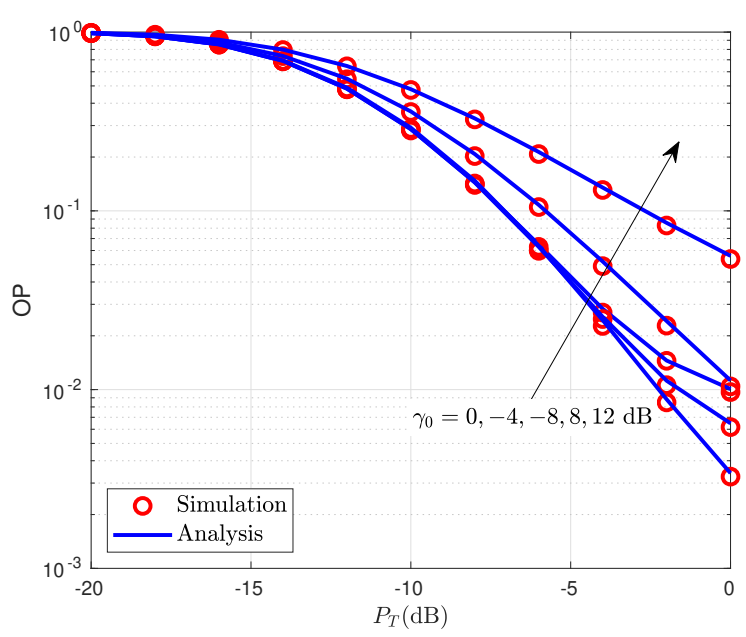

Fig. 6: OP versus $P_{T}$ for $\bar{h}_{S U}=5 \mathrm{~dB}$.

$\mathrm{dB}, N=9, \gamma_{0}=0.5 \mathrm{~dB}, \bar{h}_{T R}=3 \mathrm{~dB}, \bar{h}_{S U}=3 \mathrm{~dB}, \bar{h}_{R U}=3$ $\mathrm{dB}, d_{T R}=d_{S R}=d_{R D}=2 \mathrm{~m}, d_{S U}=3 \mathrm{~m}, d_{R U}=1 \mathrm{~m}$, $\beta=2$ and $N_{0}=1$.

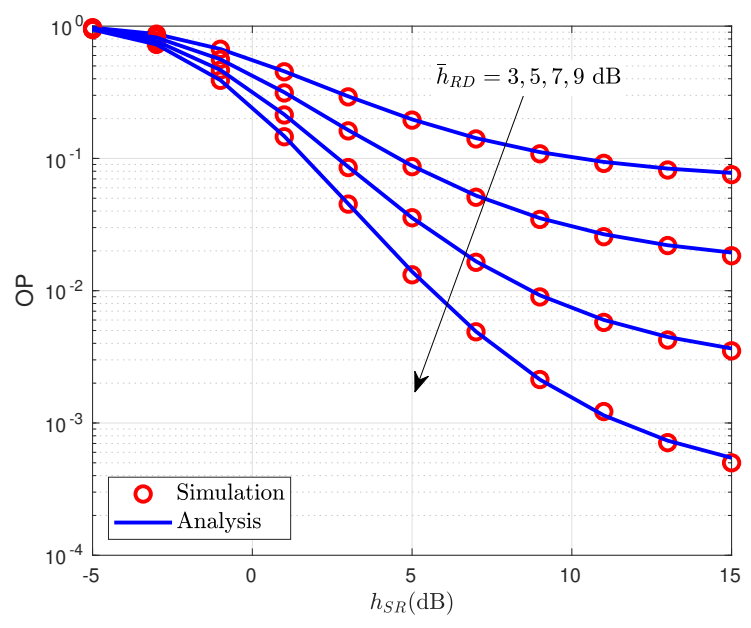

Fig. 7: OP versus $\bar{h}_{S R}$ for $\gamma_{t h}=1 \mathrm{~dB}$.

Fig. 7 illustrates that OP can be improved by increasing $\bar{h}_{R D}$ or $\bar{h}_{S R}$ link, because a high $\bar{h}_{R D}$ or $\bar{h}_{S R}$ represents a better channel condition. In the improved $S-R$ or $R-$ $D$ condition, $D$ could gain high SNR under relay selection scheme, leading to a low OP.

Fig. 8 presents that the system with a small $\gamma_{t h}$ outperforms the one with a large $\gamma_{t h}$, due to the fact that a high SNR threshold means the system need a better channel situation to communicate.

\section{Comparisons between OSRL-WEH, ORDL-WEH and OSRDL-WEH Schemes}

In this subsection, we compare the outage performance under OSRL-WEH, ORDL-WEH and OSRDL-WEH schemes. According to [5] and [25], the main adopted parameters are set as: $T_{1}=400 \mathrm{~s}, T_{2}=100 \mathrm{~s}, B_{R}=500 \mathrm{mAh} \times 3.0 \mathrm{~V}$, 


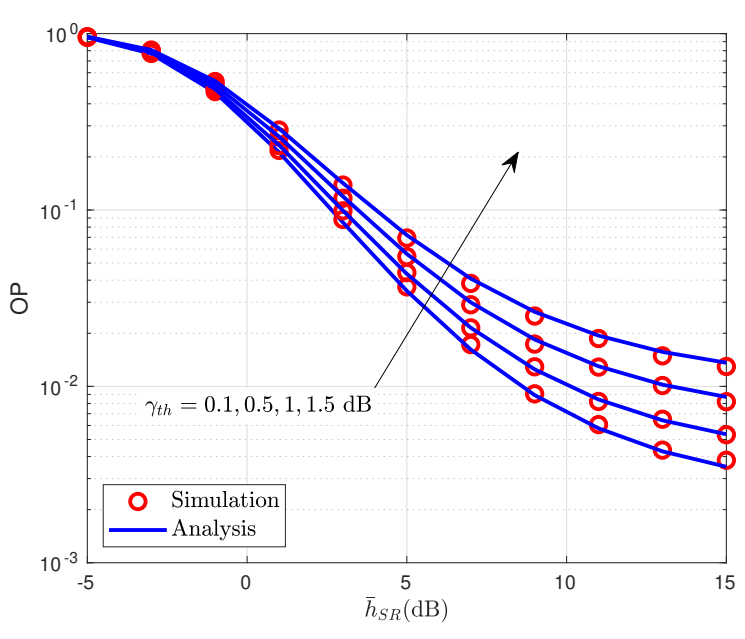

Fig. 8: OP versus $\bar{h}_{S R}$ for $\bar{h}_{R D}=6 \mathrm{~dB}$.

$P_{S}=8 \mathrm{~dB}, P=5 \mathrm{~dB}, P_{T}=8 \mathrm{~dB}, I_{0}=8 \mathrm{~dB}, \gamma_{0}=0.5 \mathrm{~dB}$, $\gamma_{t h}=1 \mathrm{~dB}, N=8, \bar{h}_{T R}=3 \mathrm{~dB}, \bar{h}_{S U}=7 \mathrm{~dB}, \bar{h}_{R U}=5$ $\mathrm{dB}, h_{S R}=7 \mathrm{~dB}, \bar{h}_{R D}=7 \mathrm{~dB}, d_{T R}=d_{S R}=d_{R U}=2 \mathrm{~m}$ $d_{S U}=d_{R D}=3 \mathrm{~m}$ and $N_{0}=1$.

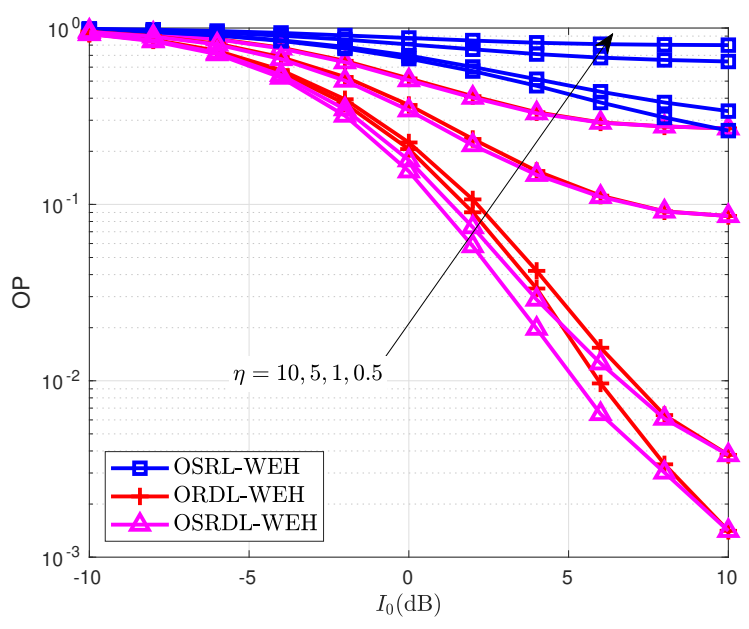

Fig. 9: OP versus $I_{0}$.

Fig. 9 plots the OP versus $I_{0}$ for various $\eta=\frac{T_{1}}{T_{2}}$ with $T_{1}=200 \mathrm{~s}$ under OSRL-WEH, ORDL-WEH and OSRDLWEH schemes, respectively. We can observe that OP can be improved by decreasing $T_{2}$ which means high transmit power over the second hop. However, there are small performance differences between ORDL-WEH and OSRDL-WEH schemes in the low $\eta$ region. The reason is that the OP for $R-D$ link will increase, leading to poor performance which dominated by $R-D$ link. Moreover, OP can be improved by increasing $I_{0}$ which means PU-RX can tolerate higher transmit power at $S$ and $R$.

Fig. 10 presents the impact of the communication distance. In other words, the influence of the pass loss will be studied. The received power at the relays decreases as $d_{T R}$ 's increasing, leading to poor $R-D$ link performance. The distance between $S$ and PU-RX does not influence the OP under

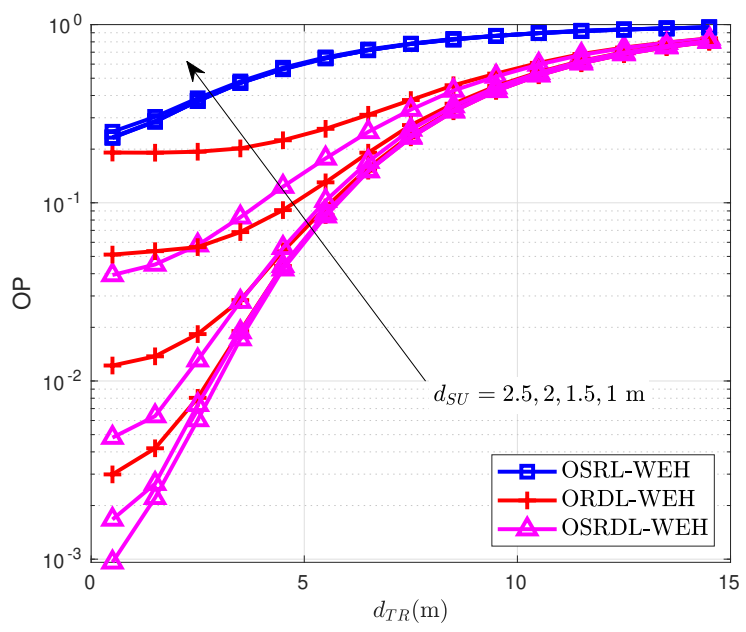

Fig. 10: OP versus $\bar{d}_{T R}$.

OSRL-WEH scheme, because of high interference tolerant capacity at PU-RX. Further distances between PU-RX and $S$ lead to higher transmit power at $S$, which can improves the performance over $S-R$ link. Moreover, there are overlaps for OSRL-WEH and OSRDL-WEH scheme, because worse OP over $R-D$ link plays a primary role in presence of the perfect outage performance over $S-R$ link.

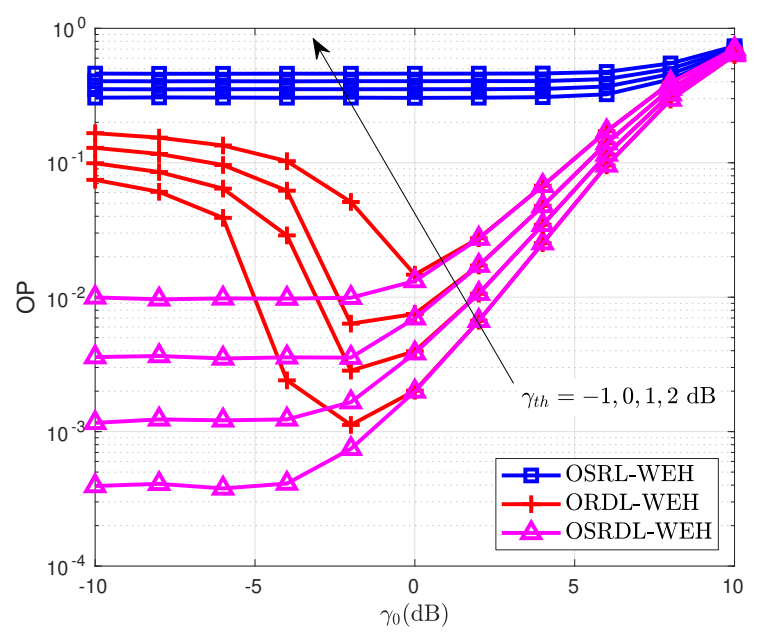

Fig. 11: OP versus $\gamma_{0}$.

As shown in Fig. 11, OP can be improved by decreasing $\gamma_{0}$ in high $\gamma_{0}$ region under OSRL-WEH and OSRDL-WEH schemes, as it can maintain more relay candidates. However, since $S-R$ link exhibits a significant contribution on the system performance, we can see that OP decreases in low $\gamma_{0}$ region and increases in high $\gamma_{0}$ region under ORDL-WEH scheme, which is similar to the findings obtained in Fig. 6 . Moreover, the OP under these three RS schemes with a small $\gamma_{t h}$ outperforms the one with a high $\gamma_{t h}$, as same as the observations achieved in Fig. 8.

Obviously, OSRDL-WEH scheme outperforms OSRL-WEH and ORDL-WEH schemes in the same condition. Because 
OSRDL-WEH scheme selects the best relay candidate for data transmission by making use of the CSI of the end-to-end $S-R_{m}-D$ link, other than only depending on the CSI of the partial link. Moreover, ORDL-WEH scheme outperforms OSRL-WEH scheme because under ORDL-WEH scheme the threshold DF scheme first choosing the relay candidates with better $S-R$ channel for best relay selection according to the quality of $R_{m}-D$ link.

\section{CONCLUSION}

This paper has investigated the outage performance of a cooperative CR system with WEH, along with considering the threshold DF relay scheme and three best RS schemes. We characterized the OP and the SNR for single $S-R_{n}$ link and $R_{m}-D$ link suffering the energy constraint at PU-RX and finite energy storage capacity. Also, we have characterized the distribution of the number of the available relay candidates under threshold relay scheme and derived the closed-from analytical expressions for the OP under OSRL-WEH, ORDLWEH and OSRDL-WEH schemes, respectively. The validity of the proposed analytical models has been verified through Monte-Carlo simulations.

By observing the numerical results, we can reach the following conclusions:

1) The threshold DF scheme could improve the system outage performance by increasing $\gamma_{0}$ in low $\gamma_{0}$ region or by decreasing $\gamma_{0}$ in high $\gamma_{0}$ region.

2) The increasing of the number of relay candidates could improve the system performance and diversity gain obviously.

3) There is a low bound of the OPs under OSRL-WEH and ORDL-WEH schemes since the quality of the other link decide the final overall OP in better channel condition.

4) ORDL-WEH scheme outperforms OSRL-WEH scheme, while OSRDL-WEH outperforms ORDL-WEH scheme and OSRL-WEH scheme, due to the exploitations of the CSI of $S-R_{m}$ and $R_{m}-D$ link.

5) The transmission time for the second hop makes little sense on the OP under ORDL-WEH scheme, while the channel condition of $S$ to PU-RX link does not influence the OP a lot under OSRL-WEH scheme.

6) The influence of the threshold DF relay scheme will degrade, while the transmission quality over the first hop being considered in the relay selection process.

7) The pass loss will decrease the harvested energy at the relays leading to bad $R-D$ link performance, as well as the low received signal power at PU-RX, which allows a high transmit power at the source and relays.

\section{REFERENCES}

[1] Z. Hasan, H. Boostanimehr, and V. Bhargava, "Green cellular networks: A survey, some research issues and challenges," IEEE Commun. Surveys Tuts., vol. 13, no. 4, pp. 524-540, 2011.

[2] K. Huang and V. Lau, "Enabling wireless power transfer in cellular networks: Architecture, modeling and deployment," IEEE Trans. Wireless Commun., vol. 13, no. 2, pp. 902-912, Feb. 2014.

[3] A. Siddiqui, L. Musavian, S. Aissa, Q. Ni. "Performance analysis of relaying systems with fixed and energy harvesting batteries," IEEE Trans. Commun., vol. 66, no. 4, pp. 1386-1398, April 2018.
[4] G. Pan, J. Ye and Z. Ding, "Secrecy outage analysis of hybrid VLC-RF systems with light energy harvesting," in Proc. IEEE SPAWC, Sapporo, 2017, pp. 1-5.

[5] G. Pan, J. Ye and Z. Ding, "Secure hybrid VLC-RF systems with light energy harvesting," IEEE Trans. Commun., vol. 65, no. 10, pp. 4348-4359, Oct. 2017.

[6] T. Le, K. Mayaram, and T. Fiez, "Efficient far-field radio frequency energy harvesting for passively powered sensor networks," IEEE J. Solid-State Circuits., vol. 43, no. 5, pp. 1287-1302, May. 2008.

[7] G. Pan, H. Lei, Y. Yuan and Z. Ding, "Performance analysis and optimization for SWIPT wireless sensor networks," IEEE Trans. Commun., vol. 65, no. 5, pp. 2291-2302, May. 2017.

[8] J. Ye, H. Lei, Y. Liu, G. Pan, D. B. da Costa, Q. Ni, Z. Ding, "Cooperative communications with wireless energy harvesting over Nakagami- $m$ fading channels," IEEE Trans. Commun., vol. 65, no. 12, pp. 5149-5164, Dec. 2017.

[9] Q. Ni, C. Zarakovitis. "Nash bargaining game theoretic scheduling for joint channel and power allocation in cognitive radio systems," IEEE $J$. on Sel. Areas in Commun., vol. 30, no. 1, Jan 2012, pp. 70-81.

[10] C. Xu, M. Zheng, W. Liang, H. Yu, and Y. Liang, "Outage performance of underlay multihop cognitive relay networks with energy harvesting," IEEE Commun. Lett., vol. 20, no. 6, pp. 1148-1151, Jun. 2016.

[11] C. Zhang, D. Wang, J. Ye, H. Lei, J. Zhang, G. Pan and Q. Feng. "Secrecy outage analysis on underlay cognitive radio system with fullduplex secondary user," IEEE Access, vol. 5, pp. 25696-25705, Dec. 2017.

[12] G. Han, J. K. Zhang, and X. Mu, "Joint optimization of energy harvesting and detection threshold for energy harvesting cognitive radio networks," IEEE Access, vol. 4, pp. 7212-7222, Oct. 2016.

[13] L. Mohjazi, M. Dianati, G. K. Karagiannidis, S. Muhaidat, and M. AlQutayri, "RF-powered cognitive radio networks: Technical challenges and limitations," IEEE Commun. Mag., vol. 53, no. 4, pp. 94-100, Apr. 2015.

[14] L. Sibomana, H. Zepernick, and H. Tran, "Wireless information and power transfer in an underlay cognitive radio network," in Proc. IEEE ICSPCS, Gold Coast, QLD, 2014, pp. 1-7.

[15] C. Zhang, J. Ye, G. Pan and Z. Ding, "Cooperative Hybrid VLC-RF Systems With Spatially Random Terminals," IEEE Trans. Commun., vol. 66, no. 12, pp. 6396-6408, Dec. 2018.

[16] D. Wang, H. Yu, H. Lei, T. Li, J. Zhang, G. Pan and Y. Chen, "Outage of relay simultaneous wireless information and power transfer with GSC and finite storage in Nakagami- $m$ fading," IET Commun., vol. 11, no. 12, pp. 1871-1881, Aug. 2017.

[17] A. El Shafie, N. Al-Dhahir and R. Hamila, "A sparsity-aware cooperative protocol for cognitive radio networks with energy-harvesting primary user," IEEE Trans. Commun., vol. 63, no. 9, pp. 3118-3131, Sept. 2015.

[18] D. K. Verma, R. Y. Chang and F. Chien, "Energy-assisted decodeand-forward for energy harvesting cooperative cognitive networks," IEEE Trans. Cog. Commun. Netw., vol. 3, no. 3, pp. 328-342, Sept. 2017.

[19] Y. Liu, S. A. Mousavifar, Y. Deng, C. Leung and M. Elkashlan, "Wireless energy harvesting in a cognitive relay network," IEEE Trans. Wireless Commun., vol. 15, no. 4, pp. 2498-2508, Apr. 2016.

[20] K. Janghel and S. Prakriya, "Throughput of underlay cognitive energy harvesting relay networks with an improved time-switching protocol," IEEE Trans. Cog. Commun. Netw., vol. 4, no. 1, pp. 66-81, Mar. 2018.

[21] D. Xie, X. Lai, X. Lei and L. Fan, "Cognitive multiuser energy harvesting decode-and-forward relaying system with direct links," IEEE Access, vol. 6, pp. 5596-5606, 2018.

[22] A. Banerjee, A. Paul and S. P. Maity, "Joint power allocation and route selection for outage minimization in multihop cognitive radio networks with energy harvesting," IEEE Trans. Cog. Commun. Netw., vol. 4, no. 1, pp. 82-92, Mar. 2018.

[23] T. Le and O. Shin, "Wireless energy harvesting in cognitive radio with opportunistic relays selection," Proc. IEEE PIMRC, Hong Kong, 2015, pp. 949-953.

[24] V. Nguyen, S. Dinh-Van and O. Shin, "Opportunistic relaying with wireless energy harvesting in a cognitive radio system," Proc. IEEE WCNC, New Orleans, LA, 2015, pp. 87-92.

[25] H. Yu, D. Wang, G. Pan, R. Shi, J. Zhang and Y. Chen, "On outage of WPC system with relay selection over Nakagami- $m$ fading channels," IEEE Trans. Veh. Technol., vol. 66, no. 9, pp. 8590-8594, Sept. 2017.

[26] Y. Zhou, G. Pan, T. Li, H. Liu, C. Tang and Y. Chen, "Secrecy outage performance for partial relay selection schemes in cooperative systems," IET Commun., vol. 9, no. 16, pp. 1980-1987, Nov. 2015.

[27] G. Pan, Y. Chen, and Q. Feng, "Performance analysis of interferencelimited cooperative systems with relay selection over independent lognormal fading channels," IET Commun., vol. 8, no. 10, pp. 1751-1761, Jul. 2014.

[28] I. S. Gradshteyn and I. M. Ryzhik, Table of Integrals, Series and Products, 6 Ed. Elsevier Pte Ltd, 2000. 
[29] A. P. Prudnikov, Yu. A. Brychkov, and O. I. Marichev, Integrals and Series: Vol. 1, Elementary Functions, 3rd Ed., New York: Gordon \& Breach Sci. Publ., 1992.

[30] A. P. Prudnikov, Y. A. Brychkov, and O. I. Marichev, Integrals and Series: Vol. 3: More Special Functions, New York: CRC Press, 1992.

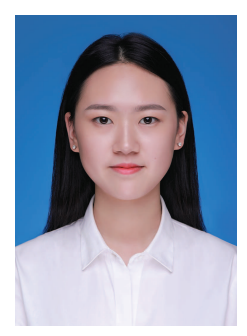

Jia Ye (S'18) received the B.Sc. degree in communication engineering from Southwest University, Chongqing, China, in 2018. Now, she is a master student at King Abdullah University of Science and Technology (KAUST), Saudi Arabia. Her main research interests include the performance analysis and modeling of wireless/wireless communication systems.

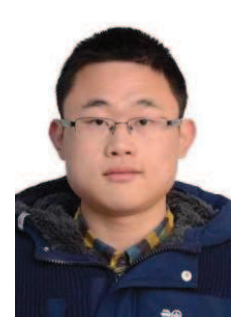

Zhedong Liu was born in Yueqing, China. He received the B.Sc. degree in Mathematics and Economics from George Mason University, Fairfax, VA,USA, in 2017 and the Bachelor of Economic$\mathrm{s}$ degree in International Economics and Trade from Nanjing University of Information Science and Technology, Nanjing, Jiangsu, China. He is now pursuing the M.Sc. degree in Statistics in King Abdullah University of Science and Technology, Thuwal, Saudi Arabia.

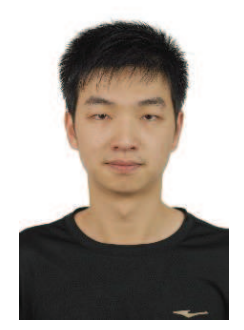

Hui Zhao (S'18) was born in Jinan, China. He received the B.Sc. degree in telecommunications engineering from Southwest University, Chongqing, China, in 2016. He is now pursuing the M.Sc. degree in electrical engineering in King Abdullah University of Science and Technology, Thuwal, Saudi Arabia.

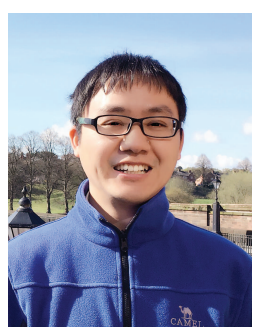

Gaofeng Pan (M'12) received his B.Sc in Communication Engineering from Zhengzhou University, Zhengzhou, China, in 2005, and the Ph.D. degree in Communication and Information Systems from Southwest Jiaotong University, Chengdu, China, in 2011.

He was with The Ohio State University, Columbus, OH, USA, from Sept. 2009 to Sept. 2011 as a joint-trained $\mathrm{PhD}$ student under the supervision of Prof. Eylem Ekici. In May 2012, he joined the School of Electronic and Information Engineering, Southwest University, Chongqing, China, where he is currently an Associate Professor. He was also with School of Computing and Communications, Lancaster University, Lancaster, U.K., from Jan. 2016 to Jan. 2018, where he was a postdoc under the supervision of Prof. Zhiguo Ding. His research interest spans special topics in communications theory, signal processing and protocol design, including visible light communications, secure communications, $\mathrm{CR} /$ cooperative communications and MAC protocols. He has served as a reviewer for major international journals, e.g., IEEE Journal of Selected Areas in Communications, IEEE Communications Magazine, IEEE Transactions on Communications, IEEE Transactions on Wireless Communications, IEEE Transactions on Signal Processing, IEEE Transactions on Vehicular Technology, etc. He received the Exemplary Reviewer Award 2017 given by IEEE Transactions on Communications.

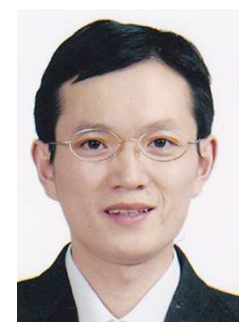

Qiang Ni (M'04-SM'08) received the B.Sc., M.Sc., and Ph.D. degrees from the Huazhong University of Science and Technology, China, all in engineering. $\mathrm{He}$ is currently a Professor and the Head of the Communication Systems Group, School of Computing and Communications, Lancaster University, Lancaster, U.K. His research interests include the area of future generation communications and networking, including green communications and networking, millimeter-wave wireless communication$\mathrm{s}$, cognitive radio network systems, non-orthogonal multiple access (NOMA), heterogeneous networks, 5G and 6G, SDN, cloud networks, energy harvesting, wireless information and power transfer, IoTs, cyber physical systems, machine learning, big data analytics, and vehicular networks. He has authored or co-authored over 200 papers in these areas. He was an IEEE 802.11 Wireless Standard Working Group Voting Member and a contributor to the IEEE Wireless Standards.

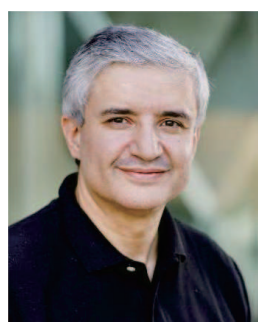

Mohamed-Slim Alouini (S'94-M'98-SM'03-F'09) was born in Tunis, Tunisia. He received the Ph.D. degree in Electrical Engineering from the California Institute of Technology (Caltech), Pasadena, CA, USA, in 1998. He served as a faculty member in the University of Minnesota, Minneapolis, MN, USA, then in the Texas A\&M University at Qatar, Education City, Doha, Qatar before joining King Abdullah University of Science and Technology (KAUST), Thuwal, Makkah Province, Saudi Arabia as a Professor of Electrical Engineering in 2009. His current research interests include the modeling, design, and performance analysis of wireless communication systems. 